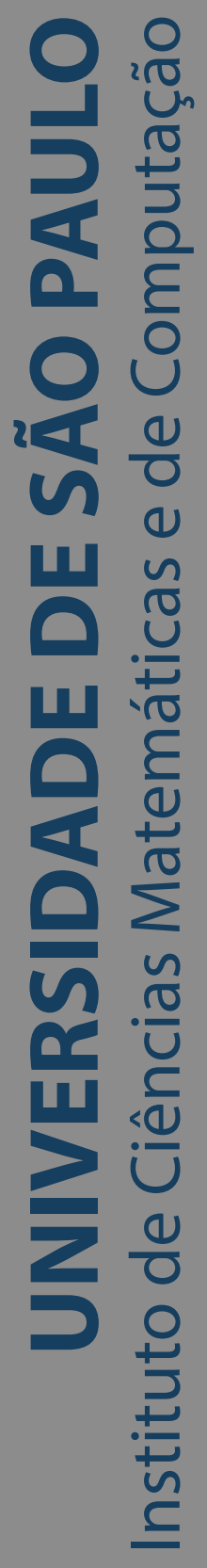

\title{
Feature extraction from 3D point clouds
}

\section{Carlos André Braile Przewodowski Filho}

Dissertação de Mestrado do Programa de Pós-Graduação em Ciências de Computação e Matemática Computacional (PPG-CCMC) 

Assinatura:

\title{
Carlos André Braile Przewodowski Filho
}

\section{Feature extraction from 3D point clouds}

\begin{abstract}
Master dissertation submitted to the Institute of Mathematics and Computer Sciences - ICMC-USP, in partial fulfillment of the requirements for the degree of the Master Program in Computer Science and Computational Mathematics. FINAL VERSION

Concentration Area: Computer Science and Computational Mathematics
\end{abstract}

Advisor: Prof. Dr. Fernando Santos Osório

\section{USP - São Carlos}

April 2018 
Ficha catalográfica elaborada pela Biblioteca Prof. Achille Bassi e Seção Técnica de Informática, ICMC/USP, com os dados inseridos pelo(a) autor(a)

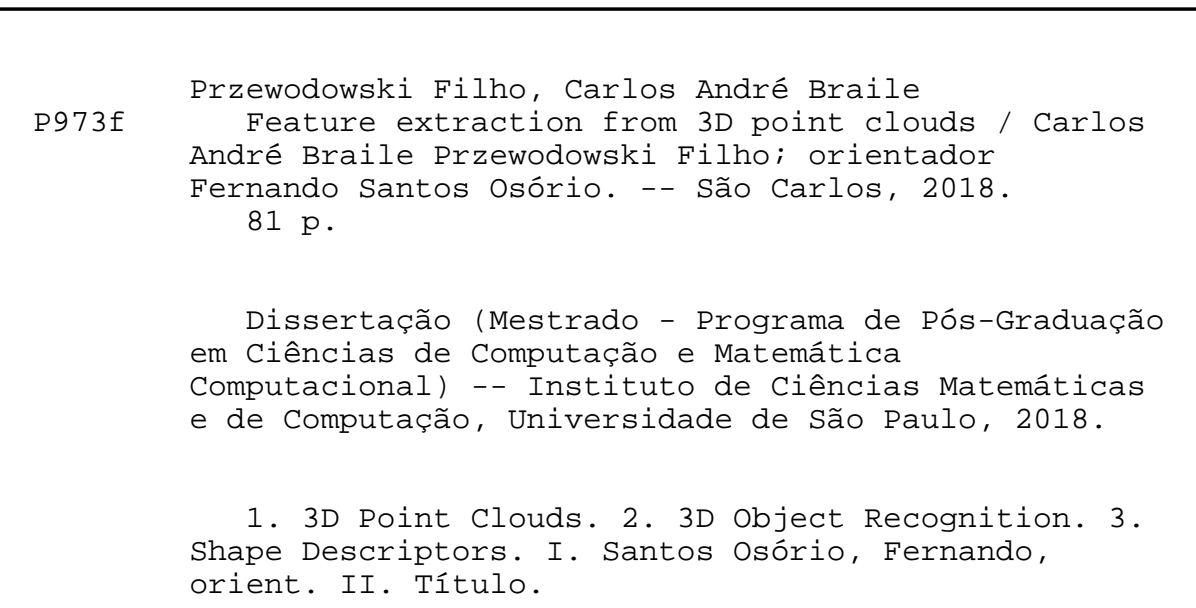

Bibliotecários responsáveis pela estrutura de catalogação da publicação de acordo com a AACR2: 
Carlos André Braile Przewodowski Filho

\title{
Extração de atributos robustos a partir de nuvens de pontos 3D
}

\author{
Dissertação apresentada ao Instituto de Ciências \\ Matemáticas e de Computação - ICMC-USP, \\ como parte dos requisitos para obtenção do título \\ de Mestre em Ciências - Ciências de Computação e \\ Matemática Computacional. VERSÃO REVISADA \\ Área de Concentração: Ciências de Computação e \\ Matemática Computacional \\ Orientador: Prof. Dr. Fernando Santos Osório
}

USP - São Carlos

Abril de 2018 

I would like to thank: CNPq and the LRM Laboratory (ICMC - USP) for their financial and material support; Professor Odemir Bruno (IFSC - USP) and Lucas Correia Ribas for their ideas and works that contributed to the development of this project; professor Luis Antônio da Silva Vasconcellos (FC - UNESP) for the help on the mathematical deductions and professor Gustavo Enrique de Almeida Prado Alves Batista (ICMC - USP) for the help on the complexity analysis of the RPHSD method. 



\section{ABSTRACT}

PRZEWODOWSKI FILHO, C. A. B. Feature extraction from 3D point clouds. 2018. 81 p. Dissertação (Mestrado em Ciências - Ciências de Computação e Matemática Computacional) Instituto de Ciências Matemáticas e de Computação, Universidade de São Paulo, São Carlos SP, 2018.

Computer vision is a research field in which images are the main object of study. One of its category of problems is shape description. Object classification is one important example of applications using shape descriptors. Usually, these processes were performed on 2D images. With the large-scale development of new technologies and the affordable price of equipment that generates 3D images, computer vision has adapted to this new scenario, expanding the classic 2D methods to 3D. However, it is important to highlight that 2D methods are mostly dependent on the variation of illumination and color, while 3D sensors provide depth, structure/3D shape and topological information beyond color. Thus, different methods of shape descriptors and robust attributes extraction were studied, from which new attribute extraction methods have been proposed and described based on 3D data. The results obtained from well known public datasets have demonstrated their efficiency and that they compete with other state-of-the-art methods in this area: the RPHSD (a method proposed in this dissertation), achieved $85.4 \%$ of accuracy on the University of Washington RGB-D dataset, being the second best accuracy on this dataset; the COMSD (another proposed method) has achieved $82.3 \%$ of accuracy, standing at the seventh position in the rank; and the CNSD (another proposed method) at the ninth position. Also, the RPHSD and COMSD methods have relatively small processing complexity, so they achieve high accuracy with low computing time.

Keywords: 3D Point Clouds, 3D Object Recognition, Shape Descriptors. 



\section{RESUMO}

PRZEWODOWSKI FILHO, C. A. B. Extração de atributos robustos a partir de nuvens de pontos 3D. 2018. 81 p. Dissertação (Mestrado em Ciências - Ciências de Computação e Matemática Computacional) - Instituto de Ciências Matemáticas e de Computação, Universidade de São Paulo, São Carlos - SP, 2018.

Visão computacional é uma área de pesquisa em que as imagens são o principal objeto de estudo. Um dos problemas abordados é o da descrição de formatos (em inglês, shapes). Classificação de objetos é um importante exemplo de aplicação que usa descritores de shapes. Classicamente, esses processos eram realizados em imagens 2D. Com o desenvolvimento em larga escala de novas tecnologias e o barateamento dos equipamentos que geram imagens $3 \mathrm{D}$, a visão computacional se adaptou para este novo cenário, expandindo os métodos 2D clássicos para 3D. Entretanto, estes métodos são, majoritariamente, dependentes da variação de iluminação e de cor, enquanto os sensores 3D fornecem informações de profundidade, shape 3D e topologia, além da cor. Assim, foram estudados diferentes métodos de classificação de objetos e extração de atributos robustos, onde a partir destes são propostos e descritos novos métodos de extração de atributos a partir de dados 3D. Os resultados obtidos utilizando bases de dados 3D públicas conhecidas demonstraram a eficiência dos métodos propóstos e que os mesmos competem com outros métodos no estado-da-arte: o RPHSD (um dos métodos propostos) atingiu $85.4 \%$ de acurácia, sendo a segunda maior acurácia neste banco de dados; o COMSD (outro método proposto) atingiu $82.3 \%$ de acurácia, se posicionando na sétima posição do ranking; e o CNSD (outro método proposto) em nono lugar. Além disso, os métodos RPHSD têm uma complexidade de processamento relativamente baixa. Assim, eles atingem uma alta acurácia com um pequeno tempo de processamento.

Palavras-chave: Nuvens de Pontos 3D, Reconhecimento de Objetos 3D, Descritores de Formato. 

Figure 1 - From the left to the right: left camera image; right camera image; depth map. Source: the 3D Stereoscopic Photography blog, available at <https://goo.gl/aHwaeY> (last access Nov. 29 2017).

Figure 2 - Example of an RGB-D image: its RGB scene (left) and its depth values (right). Source: the University of Washington RGB-D dataset website, available at $<$ https://goo.gl/TT2w8X> (last access Nov. 29 2017). . . . . . . . . . . 26

Figure 3 - Point cloud example from a reconstructed scene. . . . . . . . . . . . 27

Figure 4 - Volumetric data inside a voxel grid. Source: Gebhardt et al. (2009). . . . . . 27

Figure 5 - Sparse voxels of the volumetric data. Source: Gebhardt et al. (2009). . . . . 28

Figure 6 - Octree voxel model over a 3D object. Source: Gebhardt et al. (2009). . . . . 28

Figure 7 - A 3D mesh of a police car from the ModelNet dataset. The figure: illustrates only the object vertices (left); illustrates the edges between the vertices (middle); illustrates the faces of the mesh (right) . . . . . . . . . . . . 28

Figure 8 - A tracing line and it's parameters. Source: Kadyrov and Petrou (2001). Copyright (c) 2001, IEEE. . . . . . . . . . . . . . . . . . . . . . . 31

Figure 9 - A 2D image and it's trace transform on a given functional $T$. Source: Kadyrov and Petrou (2001). Copyright (c) 2001, IEEE. . . . . . . . . . . . . . . 32

Figure 10 - The 3D Radon Transform. Source: Daras et al. (2004). Copyright (c) 2004,

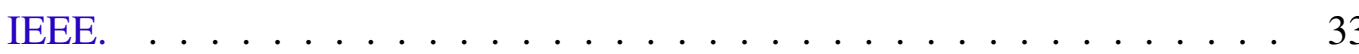

Figure 11 - Integration path of RIT. Source: Daras et al. (2006). Copyright (C) 2006, IEEE. 34

Figure 12 - Point pairs in $p$ neighbor. Source: Rusu, Blodow and Beetz (2009). Copyright (c) 2009, IEEE.

Figure 13 - The connections of FPFH for a given point $p_{q}$. The colored dots $p_{k}$ are the direct neighbors of $p_{q}$. Notice that the only connections are between $p_{q}$ and its neighbors and between $p_{k}$ and their neighbors. The lines marked with 2 will be used twice. Source: Rusu, Blodow and Beetz (2009). Copyright (c)

Figure 14 - Geometric edges detection: (a) original RGB image; (b) extracted edges using Canny detector; (c) depth image; (d) obtained geometric edges. Source: (DROST; ILIC, 2012). Copyright (c) 2013, IEEE. . . . . . . . . . . . . . . 36

Figure 15 - The range image region of the interest point. The orange lines represent the beams that cross the interest point. . . . . . . . . . . . . . . 37 
Figure 16 - VoxNet network lattice. Source: Maturana and Scherer (2015). Copyright (c) 2015, IEEE. . . . . . . . . . . . . . . . . . . . . . . 38

Figure 17 - CFK network structure. Source: Cheng et al. (2015). Copyright (C) 2015, IEEE. 39

Figure 18 - Possible transition directions for $r=1 \ldots \ldots \ldots$. . . . . . . 42

Figure 19 - Example of a horizontal co-occurrence matrix for a 2D image. Notice that there are two transitions from value 1 for 2 in the image and the COM at row 1 and column 2 has value 2. On the other hand, there are no transitions from 4 to 3 , so the COM value at row 4 and column 3 is 0 . . . . . . . . . . . 42

Figure 20 - Angles on 3D images. . . . . . . . . . . . . . . . . 43

Figure 21 - Example of an undirected graph (left) and its corresponding adjacency matrix (right) . . . . . . . . . . . . . . . . . . . . .

Figure 22 - Example of connected edges with different $T$. From the left to the right: Original network; edges when $T=0.8$; edges when $T=0.4$; edges when

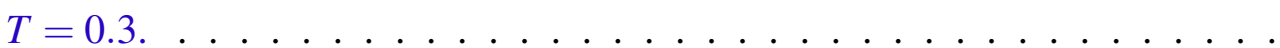

Figure 23 - Computed histograms for a box. From the left to the right: $M=50 ; M=100$; and $M=200$.

Figure 24 - On the top, three different histograms triples $(M=200)$ from: a box (left); a comb (middle); and a banana (right). On the bottom, the shapes from: a box (left); a comb (middle); and a banana (right) . . . . . . . . . . . . . . .

Figure 25 - The invariance against rotation on the center of mass (points represented on a polar coordinate system). Notice that, for any direction that the shape rotates, the points will remain at the same distance $\rho$ from the center. . . . . . . 53

Figure 26 - The points in the 3 -dimensional space. . . . . . . . . . . . . . . 54

Figure 27 - Points from the example in: (left) Cartesian coordinates; (right) polar coordinates. The blue dots are the reference points, the red dot is $P$ and the green dots are the possible $P^{\prime} \ldots \ldots \ldots \ldots \ldots$

Figure 28 - The eight reference points with angular distance $\Delta \alpha=45^{\circ}$, computed using

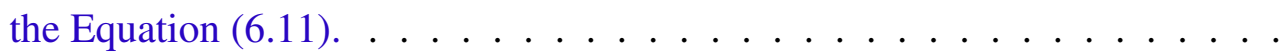

Figure 29 - Shapes of a hand towel. From the left to the right: without rotation; rotated 90 degrees; rotated 180 degrees; rotated 270 degrees. . . . . . . . . . . . .

Figure 30 - The histograms from the rotated shapes from the shape: (i) without rotation (top left); (ii) with 90 degrees rotation (top right); (iii) with 180 degrees rotation (bottom left); (iv) with 270 degrees rotation (bottom right). Notice that the histograms are the same, but swapped. For instance: $H_{1}$ from (i) is the same as $H_{2}$ from (ii), which is the same as $H_{3}$ from (iii) and $H_{4}$ from (iv).

Figure 31 - University of Washington UW RGB-D Object Dataset. On the top: object captured from UW dataset. On the bottom: subset of objects from UW dataset. Source: Lai et al. (2011). Copyright (c) 2011, IEEE. . . . . . . . . 64

Figure 32 - Samples from the MN-DS. Source: Wu et al. (2015). Copyright (c) 2015, IEEE. 64 
Figure 33 - Mugs represented on different data representation. From the left to the right: an UW segmented RGB-D data; 3D mesh from the MN model; and 3D mesh from the 3DNet train set. . . . . . . . . . . . . . . . 66

Figure 34 - Differences between instances inside the class bathtub. . . . . . . . . . 66

Figure 35 - The conversion from the 3D point cloud representation of the Stanford bunny (left) to a voxel grid (right) . . . . . . . . . . . . . . . . . . 68

Figure 36 - The reference points arrangements. From the left to the right: $A_{1}$ cross; $A_{2}$ spherical; and $A_{3}$ cubic . . . . . . . . . . . . . . . . . . 70

Figure 37 - Bar chart of recent works performance for object classification on UW objects dataset. The brown bars represent the results for the proposed methods. The blue bars are the other methods results and the black lines are the deviance of each method accuracy. . . . . . . . . . . . . . . . . . . . . 72

Figure 38 - Bar chart of recent works performance for object classification on the MN dataset. The brown bars represent this work results, the blue bars are the 

Table 1 - Table of possible directions for transitions between voxels. Source: (OTHMEN; SAYADI; FNIAECH, 2013). . . . . . . . . . . . . . . . . 43

Table 2 - Each statistical feature associated with its formula. $M$ is the size of the COM $P$ and $P[r, c]$ is the value from $P$ at the row $r$ and column $c$. . . . . . . . 44

Table 3 - COMSD-3D accuracy $(\%)$ classified with kNN on the UW-DS. . . . . . . . 71

Table 4 - CNSD-3D accuracy(\%) classified with k-NN, with K equals to 1, 5 and 15 respectively on the UW-DS. Standard deviation values missing (n.d. - not defined) due to the default Weka Interface results. . . . . . . . . . . . . . 71

Table 5 - RPHSD best accuracy (\%) results for each dataset. The Descriptor Type to Arrange columns are the parameters used on the method. The $K$ column represents the $k$ parameter of the $\mathrm{kNN}$ algorithm. . . . . . . . . . . . . 72

Table 6 - Comparison between the presented methods and recent results on UW dataset. The result in blue is the best comparable result from the previous works. The results obtained in this work are in bold font. . . . . . . . . . . . . . 73

Table 7 - Comparison between this work and recent results on MN dataset. The result in blue is the best comparable result from the previous works. The results obtained in this work are in bold font. . . . . . . . . . . . . . . . . . 74 



\section{LIST OF ABBREVIATIONS AND ACRONYMS}

$R^{2} I C A \quad$ Reconstruction Independent Component Analysis

2D Two Dimensional

3D Three Dimensional

BGF Box Grid Filtering

CFK Convolutional Fischer Kernels

CNN Convolutional Neural Networks

CNPq Conselho Nacional de Desenvolvimento Científico e Tecnológico

CNSD Complex Networks Shape Descriptor

COMSD Co-Occurrence Matrices Shape Descriptor

FC Faculdade de Ciências

FK Fisher Kernel

FPFH Fast Point Feature Histograms

GPS Global Positioning System

ICMC Instituto de Ciências Matemáticas e de Computação

IFSC Instituto de Física de São Carlos

IR Infrared

kNN k-Nearest Neighbors

LIDAR Light Detection and Ranging

LRM Laboratório de Robótica Móvel

MN-DS ModelNet Dataset

MPPF Multimodal Point Pair Features

NARF Normal Aligned Radial Features

OFF Object File Format

PFH Point Feature Histograms

Pixel Picture Element

RGB-D Red, Green, Blue and Depth

RICA Reconstruction Independent Component Analysis

RIT Radial Integration Transform

RPF Random Points Removal

RPHSD Reference Points Histograms Shape Descriptor

SPFH Simplified Point Feature Histogram 

Trace Transform

UNESP Universidade Estadual Paulista Júlio de Mesquita Filho

USP Universidade de São Paulo

UW-DS University of Washington Dataset

Voxel Volumetric Pixel 
INTRODUCTION ...................... 21

1.1

Goal ............................ 22

1.1.1 Main Goal. . . . . . . . . . . . . . . . . . . . . 22

1.1.2 Secondary Goals . . . . . . . . . . . . . . . . . . . 22

$1.2 \quad$ Proposed Methods . . . . . . . . . . . . . . . 23

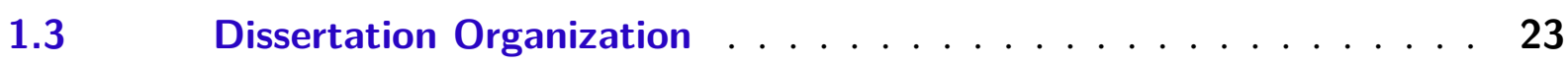

2 VOLUMETRIC IMAGES REPRESENTATION . . . . . . . . 25

$2.1 \quad$ Depth Maps and RGB-D Images . . . . . . . . . . . . . . 25

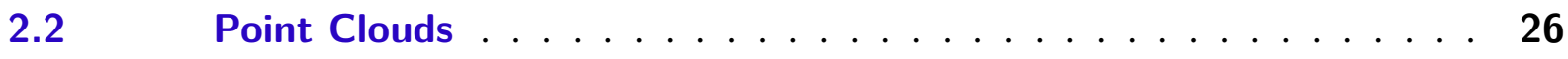

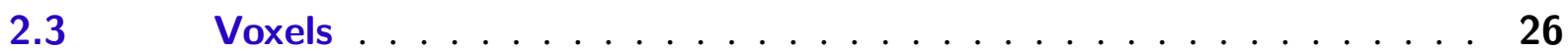

2.3.1 Gridded Voxel Model . . . . . . . . . . . . . . . . . . . . 27

2.3.2 Sparse Voxel Model . . . . . . . . . . . . . . . . . . . . 27

2.3.3 Octree Voxel Model . . . . . . . . . . . . . . . . . 27

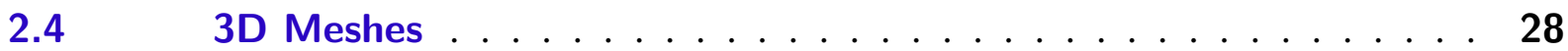

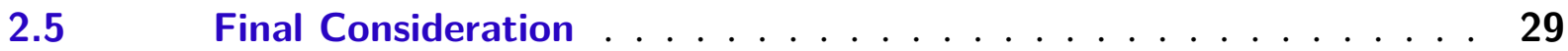

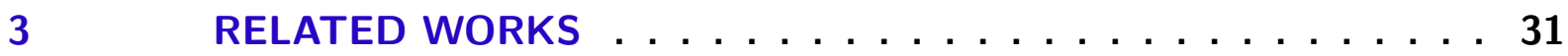

$3.1 \quad$ Trace Transforms $\ldots \ldots \ldots \ldots \ldots \ldots \ldots \ldots$

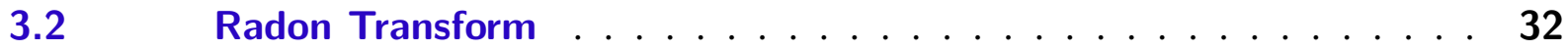

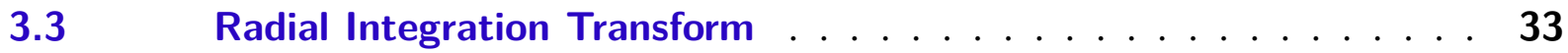

$3.4 \quad$ Fast Point Feature Histograms Descriptor . . . . . . . . . . . . 34

$3.5 \quad$ Multimodal Point Pair Features . . . . . . . . . . . . . . 36

$3.6 \quad$ Normal Aligned Radial Feature . . . . . . . . . . . . . . . . . 37

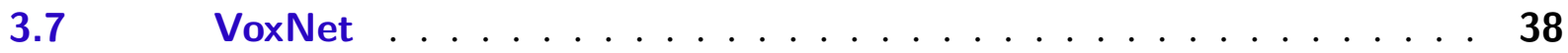

$3.8 \quad$ Convolutional Fischer Kernels . . . . . . . . . . . . . . . . . . 39

3.9 Reconstruction Independent Component Analysis

Network . . . . . . . . . . . . . . . . . . . . . . . 39

$3.10 \quad$ Fuss Convolutional Neural Networks . . . . . . . . . . . . . . . 40

3.11 Chapter Considerations . . . . . . . . . . . . . . 40

4 CO-OCCURRENCE MATRICES SHAPE DESCRIPTOR 3D . . . 41

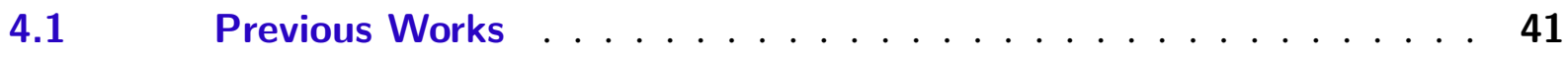

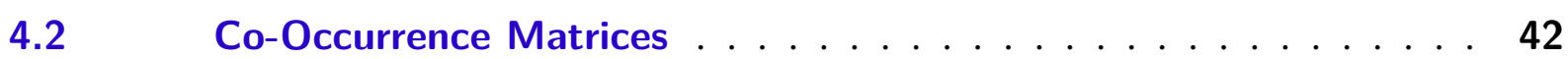


$4.3 \quad$ Features Extraction . . . . . . . . . . . . . . . 44

$4.4 \quad$ Shape Descriptor . . . . . . . . . . . . . . . 44

5 3D COMPLEX NETWORK SHAPE DESCRIPTOR . . . . . . . . 47

$5.1 \quad$ Related Works . . . . . . . . . . . . . . . . . . 47

$5.2 \quad$ Complex Networks Representation . . . . . . . . . . . . . . 48

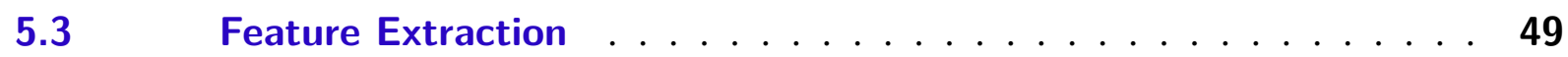

6 REFERENCE POINTS HISTOGRAMS SHAPE DESCRIPTOR . . . 51

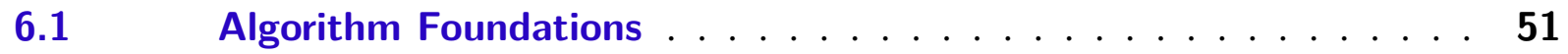

$6.2 \quad$ Reference Points Selection . . . . . . . . . . . . . . 52

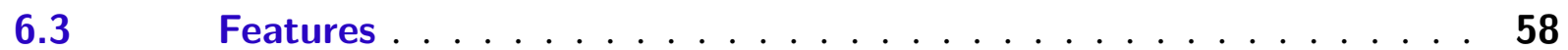

$6.4 \quad$ Algorithm and Analysis . . . . . . . . . . . . . . 59

7 DATASETS . . . . . . . . . . . . . . 63

$7.1 \quad$ University of Washington RGB-D Dataset . . . . . . . . . 63

$7.2 \quad$ Princeton's ModelNet Dataset . . . . . . . . . . . . . . . 64

$7.3 \quad$ 3DNet ..................... 65

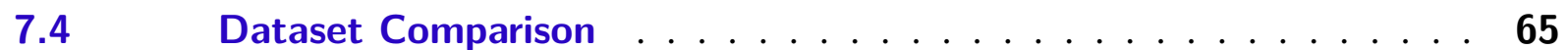

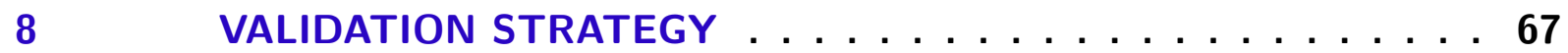

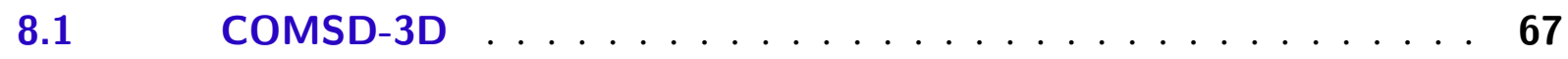

8.1.1 Dataset Setup . . . . . . . . . . . . . . . . . 67

8.1.2 Defined Parameters . . . . . . . . . . . . . . . . . 68

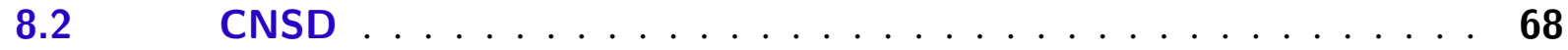

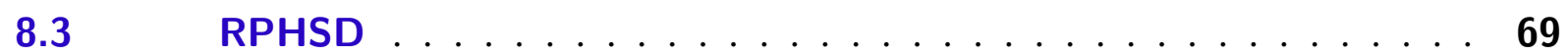

8.3.1 Dataset Setup . . . . . . . . . . . . . . . . . 69

8.3.2 Defined Parameters . . . . . . . . . . . . . . . . . . . 70

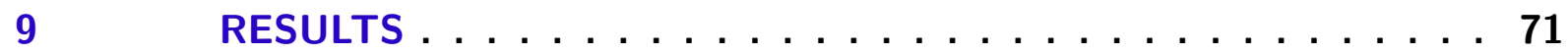

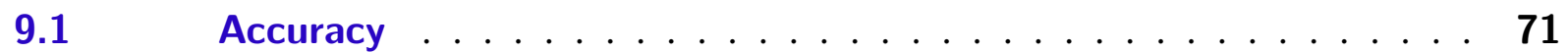

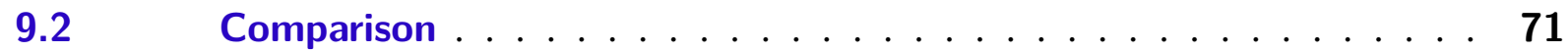

$9.3 \quad$ Results Analysis . . . . . . . . . . . . . . . . 73

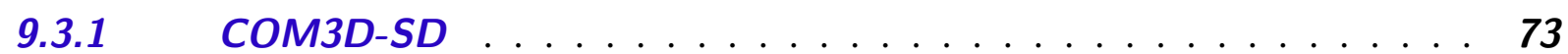

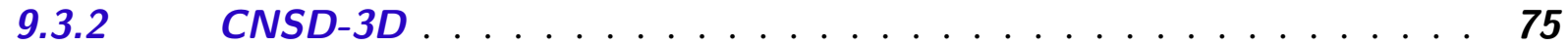

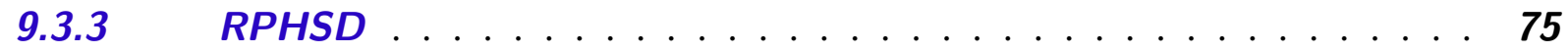

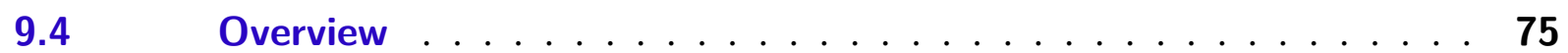

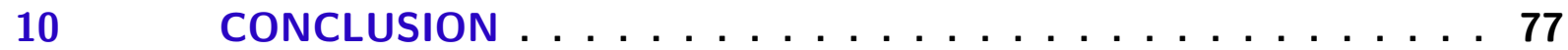

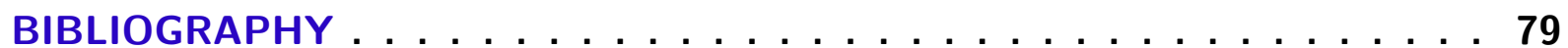


CHAPTER

1

INTRODUCTION

In the mobile robotics field, where mobile robots and autonomous intelligent vehicles are developed, the environment mapping, location determination and the detection of objects and obstacles are fundamental tasks. The mapping, localization and detection are made using several different sensors and there are many different methods and techniques for every situation. Some of these sensors enable the creation of maps of the environment from images and/or point clouds in Two Dimensional (2D) or Three Dimensional (3D) representations.

Recently, one of the most adopted sensors is the video camera, which usually provides 2D data (bitmaps representing the acquired images). However, newer sensors are coming up and are becoming popular in robotic applications, as Light Detection and Ranging (LIDAR) laser sensors and depth sensors, as, for instance, stereo cameras, Microsoft Kinect, Intel RealSense, among others, which allow to obtain 3D data (as points of $(\mathrm{x}, \mathrm{y}, \mathrm{z})$ coordinates in respect to the sensor). Considering the 2D devices, for instance monocular cameras, specific techniques for obstacle and objects identification have been developed, as for street signs detection, traffic lights, navigable regions, obstacles along the way, among many other environment elements. However, these identification techniques of $2 \mathrm{D}$ in-scene images exhibit some important issues:

- Variations in illumination (shadows, direct sun light rays incidence at the camera lens, low scene illumination);

- Reflections or surfaces that reflect light differently (for instance mirrors, water slides);

- Difficulty in estimating the distance of objects from the sensor;

- Presence of illustrations and photos of objects at the environment instead of the real object in the $3 \mathrm{D}$ world.

All these factors lead to imprecision and confusion at the scene analysis results using monocular cameras. 
On the other hand, 3D perception techniques for objects detection are usually more robust because they allow to extract a more complete description of the objects of the scene, determining their dimensions and distance to the sensor. Furthermore, some sensors are active (they emit signals and wait for their response) and do not depend on an external illumination as the conventional cameras. Like other sensors, they also depend - in a way - on the complete vision of the object, where the occlusion of elements scene can be a problem. Hence, it is important to, even from a partial vision, characterize - preferably in an individual manner - the objects from the scene. Hence, it is important to identify specific attributes of the elements of the scene that characterize them and that are invariant to position, scale, rotation (within certain limits), and also in relation to external illumination.

Identification of objects from their own attributes (descriptors or features), obtained from 3D sensors, and that are robust for variations of position, scale, rotation and illumination has a great range of applications, such as: (i) identification of milestones in the environment (landmarks) that can serve as reference for robotic location, mapping and navigation; (ii) detection and recognition of specific objects in the scene, such as obstacles; (iii) tracking an object in the scene, previously identified, which allows - for example - the creation of convoys (following a vehicle) in the case of autonomous vehicles. These applications can be focused on the development of autonomous vehicles, but also on several other applications related to mobile robotics and intelligent robotics.

Concluding, this work context is in the development of methods for extracting features on 3D shapes and 3D object recognition.

\subsection{Goal}

\subsubsection{Main Goal}

To develop robust methods that extract shape features from 3D data.

\subsubsection{Secondary Goals}

- Investigate previous shape descriptor methods and, based on them, propose new methods to extract robust features from data obtained by 3D sensors;

- Implement the methods and verify the qualities and the robustness of the extracted features;

- Evaluate the performance of the proposed methods on the task of object classification using known and available datasets. 


\subsection{Proposed Methods}

Under the development of this project, three methods were deployed: (i) the 3D CoOccurrence Matrices Shape Descriptor (COMSD); (ii) the 3D Complex Networks Shape Descriptor (CNSD); and (iii) the Reference Points Histograms Shape Descriptor (RPHSD).

The COMSD-3D is an application of the classical co-occurrence matrices for shape description and the CNSD-3D is an expansion from the 2D concept of Complex Networks to a 3D context.

The RPHSD is a novel method that basically receives a set of previously selected reference points; computes for each reference point a histogram of its distance to the point cloud points; from those histograms, several statistical features are computed and stored in a descriptor.

The source code of this project methods can be found at GitHub ${ }^{1}$ and BitBucket ${ }^{2}$.

\subsection{Dissertation Organization}

The dissertation was organized as follows:

- Chapters 2 and 3 provide some concepts and methods to understand the proposed methods;

- Chapters 4 to 6 presents the proposed methods;

- Chapter 7 introduces the datasets used to test the methods;

- Chapters 8 and 9 provide detailed explanation of the tests, the results and a comparison with other methods;

- Chapter 10 concludes this work and presents possibilities for further research.

At $<$ https://github.com/cabraile $>$

2 At $<$ https://bitbucket.org/carlos_braile/> 

CHAPTER

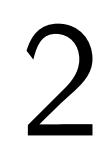

(1)

\section{VOLUMETRIC IMAGES REPRESENTATION}

There are different ways of representing 3D information: they can either be represented by non-metric data structures, as depth maps and RGB-D images, or by metric structures, for instance point clouds, 3D occupancy grids and voxels. In this chapter these data structures are presented and discussed.

\subsection{Depth Maps and RGB-D Images}

Depth maps are 2D images in which each Picture Element (Pixel) represents an acquired depth value in respect to the sensor. An usual sensor employed to generate depth maps is the stereo camera. Figure 1 illustrates a depth map generated from a stereo camera.

Red, Green, Blue and Depth (RGB-D) images are depth maps with color information. They are a 2D matrix structure that store at each pair of rows and columns the pixel color information (red, green blue) and depth value of the scene. A popular sensor used to capture RGB-D images is the Kinect Sensor from Microsoft Company (device used together with the XBox 360 and XBox One game consoles). Basically, a Kinect projects an Infrared (IR) pattern to the environment. The environment reflects the IR pattern - deformed - to the Kinect and it computes the depth based on the deformation of the pattern. An RGB-D scene was illustrated at
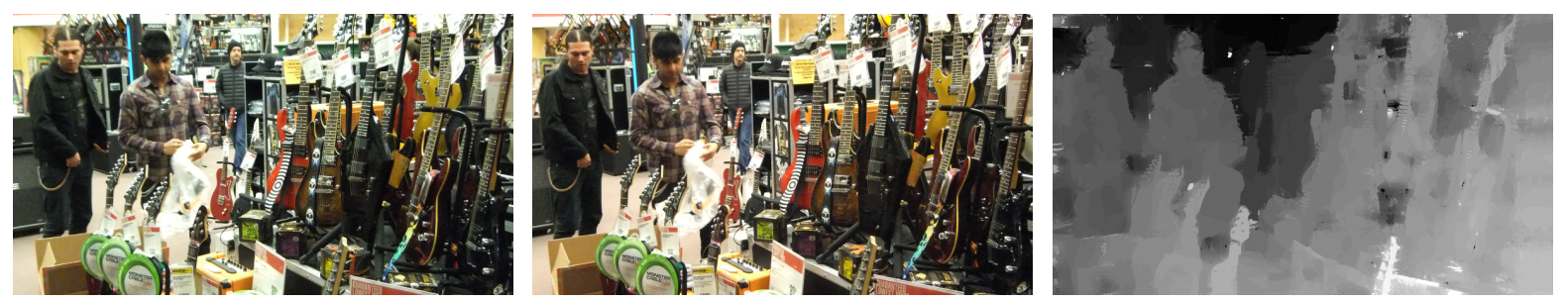

Figure 1 - From the left to the right: left camera image; right camera image; depth map. Source: the 3D Stereoscopic Photography blog, available at <https://goo.gl/aHwaeY> (last access Nov. 29 2017). 

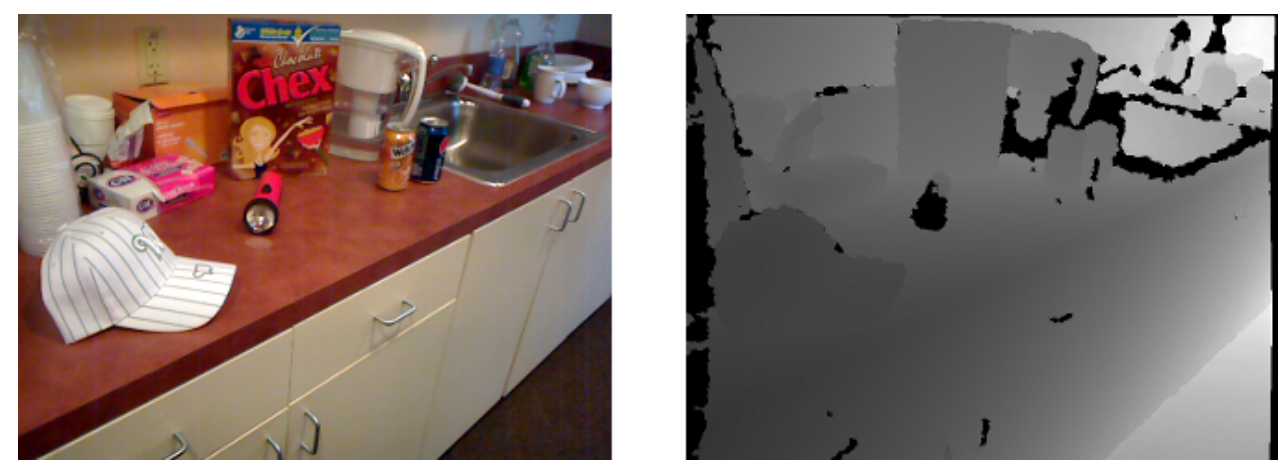

Figure 2 - Example of an RGB-D image: its RGB scene (left) and its depth values (right). Source: the University of Washington RGB-D dataset website, available at <https://goo.gl/TT2w8X> (last access Nov. 29 2017).

Figure 2. Notice that, at some regions of the scene, the depth information has noise. It happens because of how the scene objects reflect the IR pattern.

\subsection{Point Clouds}

A 3D point cloud (illustrated in Figure 3), is a set of points distributed in three geometric coordinates (XYZ), usually respecting the dimension and proportion of the objects and the environment they are located in (metric representation from 3D space). Furthermore, the represented points can have color information. There are two types of point clouds: (i) organized point clouds and (ii) unorganized point clouds.

Type (i) clouds have their dimensions (height and width) defined and are ordered by a matrix-like structure, where the data are accessed by rows and columns. One can notice that both disparity maps and RGB-D images are types of organized point cloud. The advantage from (i) is: knowing the position of adjacent points, the computational cost of many algorithms is reduced. However, as in $2.5 \mathrm{D}$ images, they are viewpoint dependent, leading to the loss of data from the occluded part of the scene.

The clouds of type (ii) are a list of points that are not organized in space and, usually, their coordinates are represented by floating-point values. Unlike type (i) clouds, there may be points without a neighbor within a determined distance, since there is no direct or implicit relation between the points.

\subsection{Voxels}

Volumetric Pixel (Voxel) is a pixel equivalent in 3D and can be stored in many different structures. In this section we present the three more usual models to represent voxels (GEBHARDT et al., 2009): the gridded, the sparse and the octree voxel models. They tend to approach the $3 \mathrm{D}$ computer vision problem to $2 \mathrm{D}$ problems, which already have a very consolidated base 


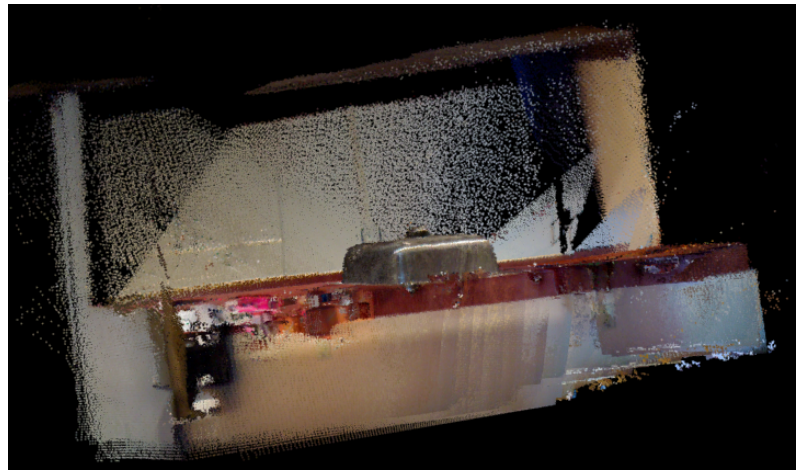

Figure 3 - Point cloud example from a reconstructed scene.

of methods and algorithms.

\subsubsection{Gridded Voxel Model}

The gridded voxel models represent the volumetric data in a grid of voxels (Figure 4). They are, for 3D images, the analog of bitmaps, from 2D images representation. The advantage of this model is its ability to randomly each element in the same way that in a $2 \mathrm{D}$ image, allowing application of classic image processing and computer vision techniques on volumetric data. On the other hand, it uses a larger amount of memory and the application of any $2 \mathrm{D}$ technique would have to take in account elements from one more dimension, which lead to higher algorithm complexity.

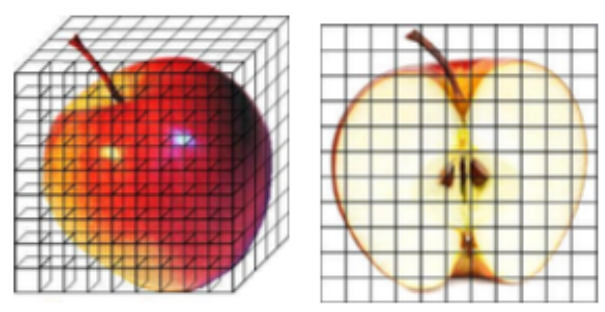

Figure 4 - Volumetric data inside a voxel grid. Source: Gebhardt et al. (2009).

\subsubsection{Sparse Voxel Model}

It also represents volumetric data through a grid. However, cells from the grid that don't have information are not considered. The advantage of this model is that it only stores existing volumetric data, reducing the amount of processed data in case of 3D data with few points. In practical aspects, the sparse voxel model is not so different than the point cloud approach.

\subsubsection{Octree Voxel Model}

Octree is a tree-based data structure in which every internal node has eight children. Regarding 3D images, an octree divides the image into eight octants. If any octant contains a 

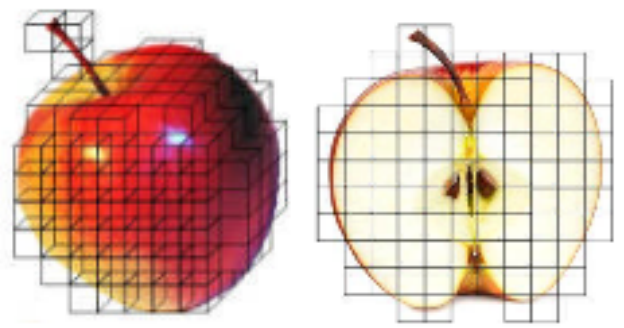

Figure 5 - Sparse voxels of the volumetric data. Source: Gebhardt et al. (2009).

voxel, it is divided into eight more octants. This is repeated recursively until a certain defined depth is achieved.

That way, the octree keeps the grid of 3D data, but reduces drastically the size of the structure, leading to lower time complexity to iterate over the whole structure and lower memory consumption. Figure 6 is an illustration of a 3D object represented as an octree voxel model.
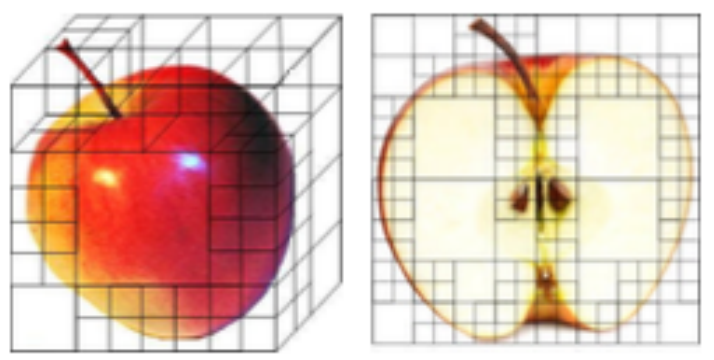

Figure 6 - Octree voxel model over a 3D object. Source: Gebhardt et al. (2009).

\subsection{D Meshes}

3D meshes are structures that contain a list of vertices (analogous to $X Y Z$ points), faces (a list of vertices set that represent a polygonal face) and edges (connections between vertices). They can be generated, for instance, by a 3D modelling software. Figure 7 illustrate these components from a 3D mesh.
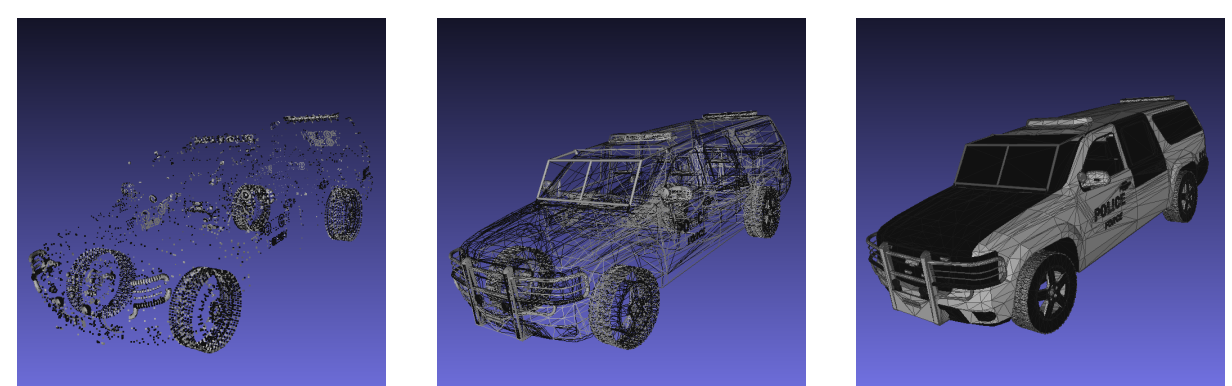

Figure 7 - A 3D mesh of a police car from the ModelNet dataset. The figure: illustrates only the object vertices (left); illustrates the edges between the vertices (middle); illustrates the faces of the mesh (right). 


\subsection{Final Consideration}

A scene representation can be made in a $2 \mathrm{D}$ image or through tridimensional representation, as presented in this chapter. The adopted representation will have a significant impact on the algorithms and in the data handling, and - furthermore - can contribute (or interfere) at the adaptation of classic algorithms adopted in 2D images that can be studied and implemented in 3D spaces. 

CHAPTER

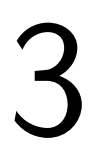

RELATED WORKS

This chapter provides a survey of related algorithms for 3D shape description and/or 3D objects classification. It starts with transform based methods - Trace Transform (TT), Radon Transform and Radial Integration Transform (RIT) - followed by the Fast Point Feature Histograms (FPFH) descriptor, the Multimodal Point Pair Features (MPPF), the Normal Aligned Radial Features (NARF) and Deep Learning approaches - VoxNet, Convolutional Fischer Kernels (CFK), Reconstruction Independent Component Analysis $\left(R^{2} I C A\right)$ network and Fuss-CNN. Finally, it ends with a final consideration, discussing the presented approaches.

\subsection{Trace Transforms}

A Trace Transform consists in the projection of the image on the hyperplanes which are normal to the center of the image with $\phi$ angle and $\rho$ distance. On 2D images, the TT projection occurs on a line perpendicular to the radius of the central point (Figure 8).

The values obtained of the image $F(C ; \phi, \rho, t)$ along each line $L(C ; \phi, \rho)$ can be inter-

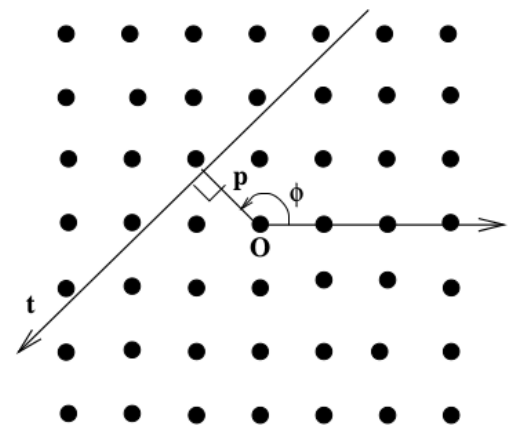

Figure 8 - A tracing line and it's parameters. Source: Kadyrov and Petrou (2001). Copyright (c) 2001, IEEE. 

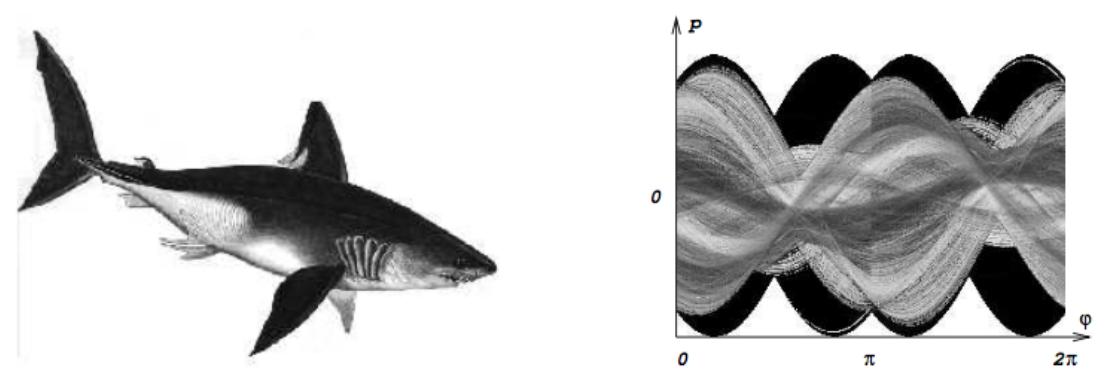

Figure 9 - A 2D image and it's trace transform on a given functional $T$. Source: Kadyrov and Petrou (2001). Copyright (c) 2001, IEEE.

preted as another image defined on $\Lambda$, as shown in Figure 9. $C$ is the coordinate system of the image; and $\Lambda$ is the set of all lines of the image.

The TT $g$ is defined as

$$
g(F ; C ; \phi, \rho)=T(F(C ; \phi, \rho)),
$$

where $T$ is the trace functional (a functional maps a vector from a vector space to a scalar space). The functional is selected depending on the application and for each application, the functional changes. A set of functionals and their application can be found on Kadyrov and Petrou (2001).

The trace transform concept is the base of the Radon Transform and the Spherical Trace Transform Descriptor, as both are described on the following subsections.

\subsection{Radon Transform}

The Radon transform is a special kind of trace transform, in which the functional is $R$ (KADYROV; PETROU, 2001), such that:

$$
R(\eta, \rho)=\int_{x \in \Pi(\eta, \rho)} f(x) d x
$$

where $\Pi$ is the plane perpendicular to the origin with direction $\eta$ and distance $\rho$, and $x$ is an image point. On another words, the Radon transform sums all the points of the image which belongs to the hyperplane (Figure 10).

The Equation (3.2) can be rewritten using the Dirac's delta function $\delta($.$) (DARAS et al.,$ 2004):

$$
R(\eta, \rho)=\int_{-\infty}^{+\infty} f(x) \delta\left(x^{T} \eta-\rho\right) d x
$$

The function $\delta($.$) is knows as:$

$$
\delta(t)=\left\{\begin{array}{ll}
0, & t \neq 0 \\
1, & t=0
\end{array},\right.
$$




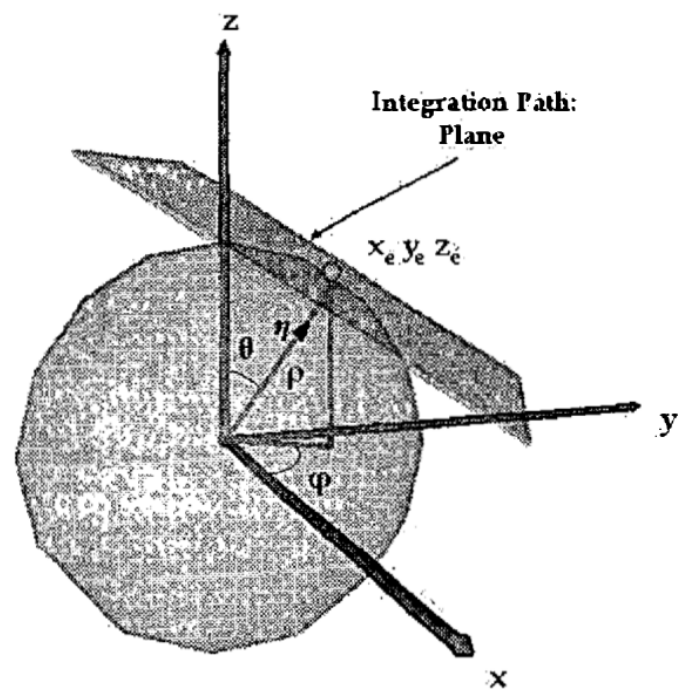

Figure 10 - The 3D Radon Transform. Source: Daras et al. (2004). Copyright (c) 2004, IEEE.

which still means that, if the distance of the image point to the hyperplane is 0 , then it belongs to the plane, thus it is summed with the other points.

The discrete form of the Radon transform (on Dirac's delta notation) is

$$
R\left(\eta_{i}, \rho_{i}\right)=\sum_{j=1}^{J} f\left(x_{j}\right) \delta\left(x_{j}^{T} \eta_{i}-\rho_{i}\right) .
$$

where $i=1, \cdots, N_{R}, N_{R}$ is the total number of planes and $J$ is the total number of points $x_{j}$.

\subsection{Radial Integration Transform}

The RIT (DARAS et al., 2006) works like the Radon Transform, but instead of projecting points to a hyperplane, it projects them onto the line segment that passes through the origin $L$ (Figure 11), such as $L(\eta, \rho)=\left\{x\left|\frac{x}{|x|}=\eta, \rho \leq\right| x \mid<\rho+\Delta_{\rho}\right\}$ and has length of $\Delta_{\rho}$.

$$
R I T(\eta, \rho)=\int_{x \in L(\eta, \rho)} f(x) d x
$$

The Equation (3.6) can be rewritten using the Dirac's delta function and the unit step function $u($.$) :$

$$
\operatorname{RIT}(\eta, \rho)=\int_{-\infty}^{+\infty} f(x) \delta\left(\frac{x}{|x|}-\eta\right) d x
$$

The discrete form of the RIT transform (on Dirac's delta notation) is

$$
\operatorname{RIT}\left(\eta_{i}, \rho_{i}\right)=\sum_{j=1}^{J} f\left(x_{j}\right) \cdot \delta\left(\frac{x_{j}}{\left|x_{j}\right|}-\eta_{i}\right)
$$




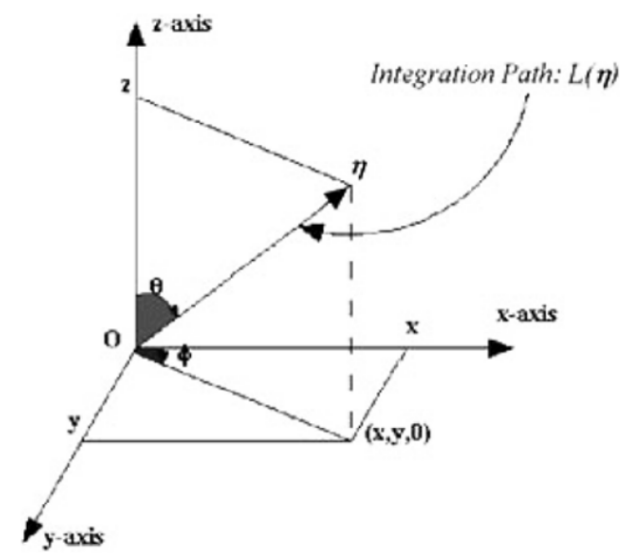

Figure 11 - Integration path of RIT. Source: Daras et al. (2006). Copyright (c) 2006, IEEE.

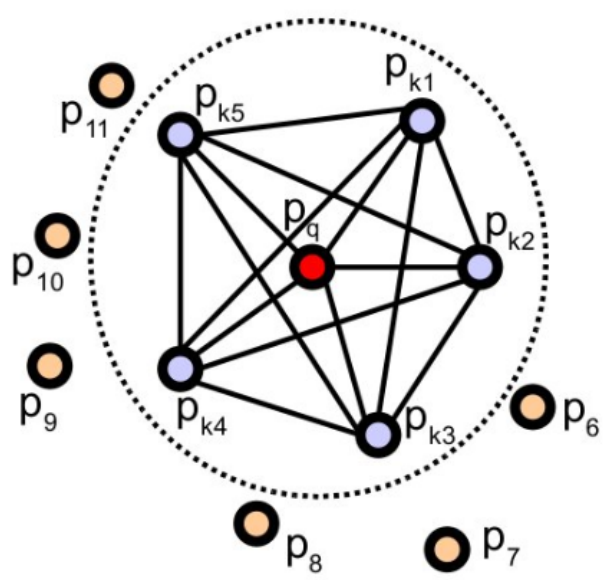

Figure 12 - Point pairs in $p$ neighbor. Source: Rusu, Blodow and Beetz (2009). Copyright (C) 2009, IEEE.

\subsection{Fast Point Feature Histograms Descriptor}

The Point Feature Histograms (PFH) describe the local geometry around a point $p$ of the point cloud. It was proposed by Rusu et al. (2008) and Rusu, Blodow and Beetz (2009) proposed improvements in its computation time, calling it as FPFH. The original PFH is pose-invariant, which is a very desirable property.

Initially, a 3D point cloud of $(x, y, z)$ coordinate points and their normals $(n x, n y, n z)$ must be provided. It is possible to use other properties instead of normals, such as curvature and $2^{\text {nd }}$ order moment invariants for instance. First the neighboring points $P_{n}$ around each point $p$ with distance $r$ are selected. After the selection, it is defined for each pair of points on $P_{n}$ (including p) a Darboux frame and the angular variations of the normals of each point pair are computed. Figure 12 represents the connections between the point pairs inside the neighboring region.

However, some shapes may have a large number of similar PFH, which may lead to ambiguous correspondences. The authors noticed that, given the mean $(\mu) \mathrm{PFH}$ of the dataset 


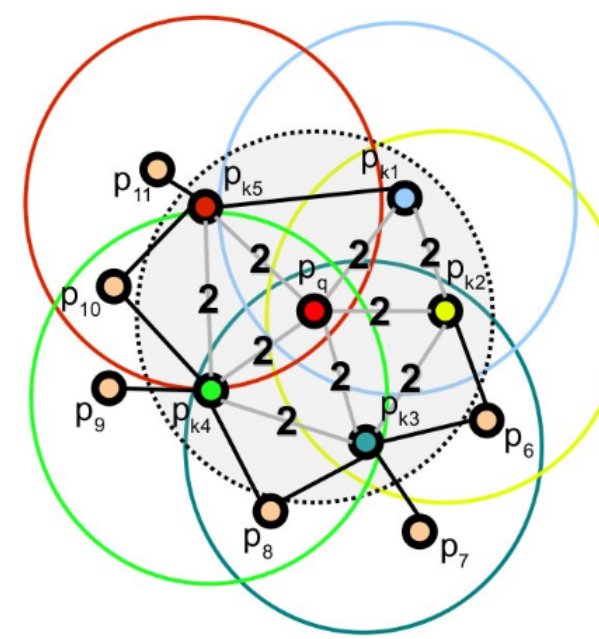

Figure 13 - The connections of FPFH for a given point $p_{q}$. The colored dots $p_{k}$ are the direct neighbors of $p_{q}$. Notice that the only connections are between $p_{q}$ and its neighbors and between $p_{k}$ and their neighbors. The lines marked with 2 will be used twice. Source: Rusu, Blodow and Beetz (2009). Copyright (c) 2009, IEEE.

and the standard deviation $(\sigma)$, the features that are outside $\mu \pm \beta \cdot \sigma$ interval are considered less common and are named "unique". The parameter $\beta$ controls the width of the interval. A point is considered persistent if it is unique in a given radius $r$ and it is unique in both $r_{i}$ and $r_{i+1}$, that is:

$$
P_{f}=\bigcup_{i=1}^{n-1} P_{f_{i}} \cap P_{f_{i+1}}
$$

where $P_{f_{i}}$ is the set of unique points from a given radius $r_{i}$.

The FPFH is a simplified version of the PFH. The first step of FPFH consists in computing only the relation between the point $p$ and it's neighbors instead of computing the relation between all the possible point pair on the neighborhood. The computed features are stored in the Simplified Point Feature Histogram (SPFH). The next step is to compute the SPFH of the neighbors $p_{n}$ of $p$ to weight the final histogram of $p$ (FPFH):

$$
F P F H(p)=\operatorname{SPFH}(p)+\frac{1}{k} \sum_{n=1}^{k} \frac{1}{w_{n}} \cdot \operatorname{SPFH}\left(p_{n}\right)
$$

where the weight $w_{n}$ is the distance between $p$ and $p_{n}$. The connection between the points are illustrated in Figure 13.

The authors have noticed that the discriminating power of PFH is slightly greater than that of FPFH. However, the computed complexity of FPFH is $O(n \cdot k)$, while the complexity of $\mathrm{PFH}$ is $O\left(n \cdot k^{2}\right)$, which means that FPFH tends to compute faster the features than PFH. 


\subsection{Multimodal Point Pair Features}

Proposed by Drost and Ilic (2012), the Multimodal Point Pair Features (MPPF) method extracts and trains a model with features extracted from RGB-D images. In this section, only the feature extraction process is detailed.

Briefly, MPPF detect edges from the 2D RGB image and filter then using the depth image to provide only the geometric edges. Then the feature vector is computed from the geometric relations between the viewpoint of the sensor and the geometric edges.

Given the RGB image from the multimodal image, a Canny edge detector extracts edges from the RGB texture. With the detected edges and their gradient direction, they are filtered considering the depth discontinuities following the gradient direction at the depth image. These steps are illustrated in Figure 14.

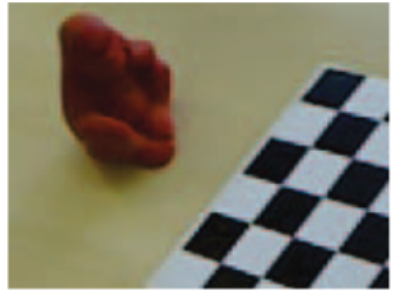

(a)

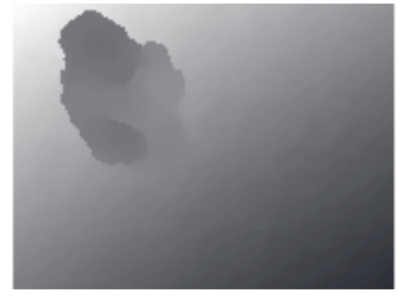

(c)

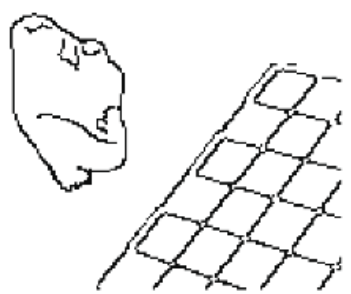

(b)

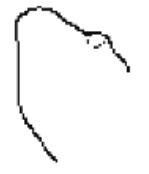

(d)

Figure 14 - Geometric edges detection: (a) original RGB image; (b) extracted edges using Canny detector; (c) depth image; (d) obtained geometric edges. Source: (DROST; ILIC, 2012). Copyright (c) 2013, IEEE.

The feature vector (iii) $F(e, r)=\left(d(e, r), \alpha_{d}, \alpha_{n}, \alpha_{v}\right)$ contains the features computed using the reference point $r$ (center of the viewpoint) and the edges $e$ :

- Metric distance $d(e, r)$ : the distance between $r$ and $e$;

- Angle $\alpha_{d}$ : the angle between the difference vector $(e-r)$ and the edge gradient

- Angle $\alpha_{n}$ : the angle between the difference vector and the normal vector of the reference point

- Angle $\alpha_{v}$ : the angle between the the normal vector and the direction towards the camera

Finally, those features are computed using other viewpoints and then a model is built to describe the scene object. 


\subsection{Normal Aligned Radial Feature}

The Normal Aligned Radial Feature (NARF) method (STEDER et al., 2010; STEDER et $a l ., 2011)$ was designed for viewpoint-dependent 3D range images, e.g. images from laser range finders. The algorithm detects borders, extracts interest points using them, and then computes the descriptor on each interest point.

Border Extraction. The NARF algorithm extracts borders from the range image. In this step, three types of points are detected: obstacle borders, if the borders actually belong to an object; shadow borders, if the border region is after the object in respect to the viewpoint; and veil points, which are interpolated points between the obstacle's borders and its "shadow". For each point from the range image, the algorithm computes the 3D distance (distance that considers the point row, column and depth values) from its neighbors. With these, considering the difference between the point position and the distance average of its neighbors, a score is computed and used to determine the type of point.

Interest Points Extraction. The interest points must, according to the authors: consider the borders and surface structure information; be detectable on different viewpoints; be on stable positions on the surface for normal estimation. This step requires the computed borders from the previous step. For the surrounding of each point, the interest value is computed, which represents the difference from the dominant direction of the other points and how the surface in the point changes. Then the interest values are smoothed and non-maximum suppression is performed to find the final interest points.

Descriptor. The NARF descriptor is computed for each interest point and depends on the pixels that belong to crossing lines (or beams) of a star pattern centered around it. This pattern is illustrated in Figure 15.

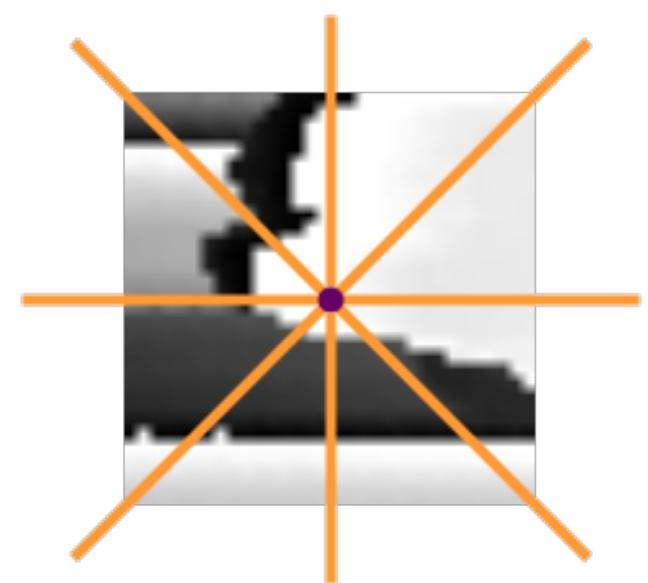

Figure 15 - The range image region of the interest point. The orange lines represent the beams that cross the interest point.

For each beam, a value that represents the changes of the pixels under it is computed and stored into a vector of size $N$, where $N$ is the number of equally spaced beams of the star 
pattern. The value of the beam is computed considering an weighted distance from each pixel that belongs to the beam in respect to the center. The descriptor can be compared other descriptors to find similar points in the scene.

\subsection{VoxNet}

VoxNet (MATURANA; SCHERER, 2015) is a convolutional network that classifies objects from point clouds. The method generates a fixed-size volumetric occupation grid with $I \times J \times K$ voxels of the image and uses it as input to the network. The output of the network indicates the probability of the input belonging to the corresponding class of each output neuron.

The network architecture consists in three stages (Figure 16): the first has only one convolutional layer; the second consists in a convolutional layer and other of pooling; the last (classification) is a densely connected network.

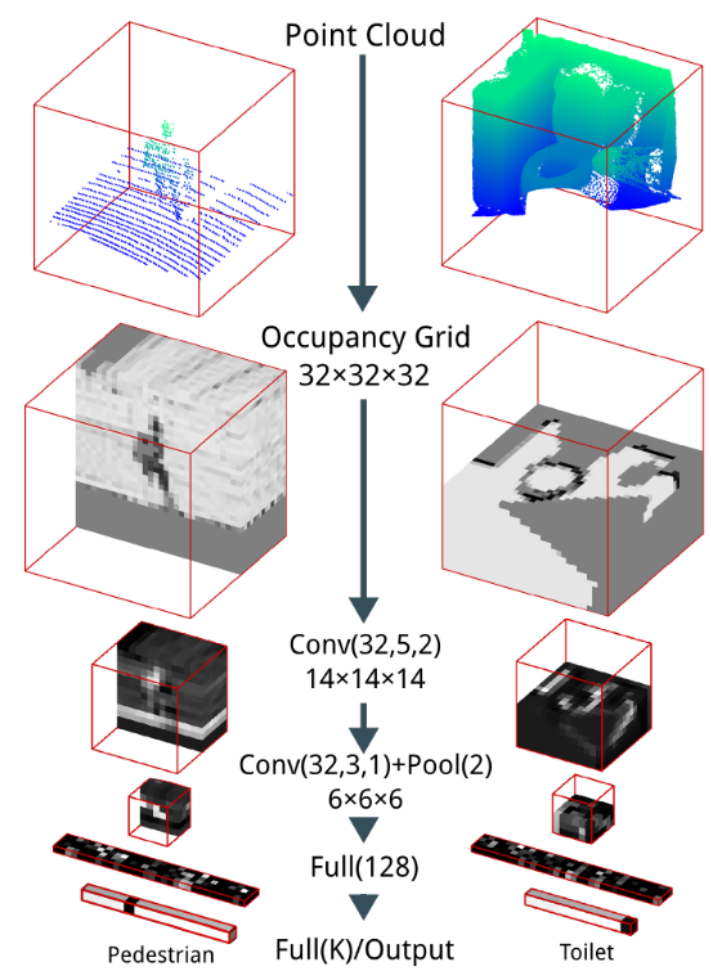

Figure 16 - VoxNet network lattice. Source: Maturana and Scherer (2015). Copyright (c) 2015, IEEE.

The convolutional layer $C(f, d, s)$ of each stage receives as input four-dimensional volumes in which three of the dimensions are spatial and the other has the characteristic map. They generate $f$ feature maps from the convolution of the layer input with $f$ filters of dimensions $d \mathrm{x} d \mathrm{x} d \mathrm{x} f^{\prime}$, where $d$ is its spatial dimension and $f^{\prime}$ is the number of feature maps. The amount of spatial displacement (or stride) of each filter is given by $s$.

The pooling layer $P(m)$ performs downsample of the input volume of the layer by a factor $m$ along the spatial dimensions by replacing each block of voxels of dimensions $m \times m \times m$ 
non-overlapped by their maximum value.

The densely connected layer $F C(n)$ has $n$ output neurons. The output of each neuron is given by the linear combination of the activation of all the neurons of the previous layer. It uses the ReLU (rectified linear unit) function $f(x)=\max \{0, x\}$, where $x$ is the linear combination of activation of all neurons of the previous layer for the activation of each neuron, with the exception of the neurons of the output layer, in which the non-linear activation function is used, providing stochastic output.

\subsection{Convolutional Fischer Kernels}

The Convolutional Fischer Kernels method was proposed by Cheng et al. (2015). It integrates the Convolutional Neural Networks (CNN) concept with the Fisher Kernel (FK) encoding. According to the authors, it unites the flexibility of the CNNs and the encoding capacity with small training data of the FK.

It basically has two layers: a single layer CNN that extracts low-level features from RGB-D data; and a FK layer that encodes the first layer. The network structure is represented at Figure 17.

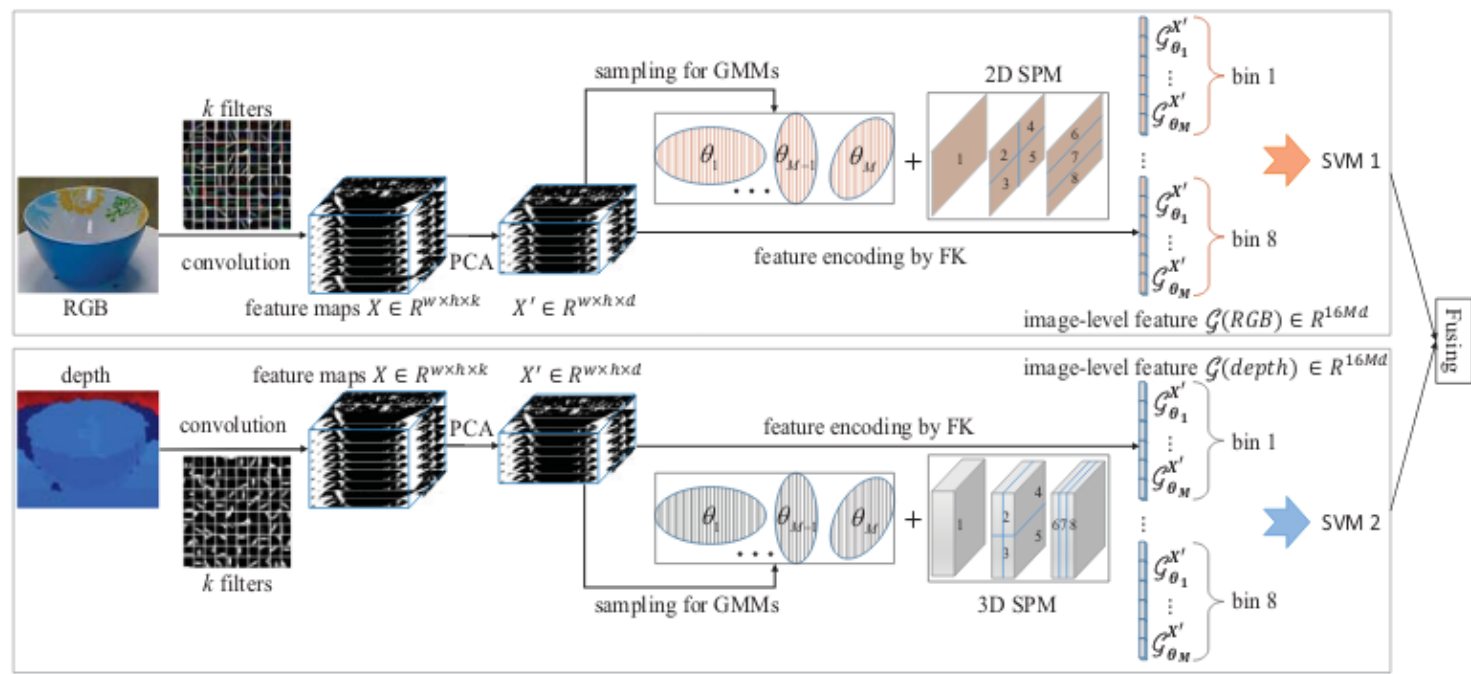

Figure 17 - CFK network structure. Source: Cheng et al. (2015). Copyright (C) 2015, IEEE.

\subsection{Reconstruction Independent Component Analysis Network}

The Reconstruction Independent Component Analysis network (JHUO et al., 2015), is a deep neural network that uses the Reconstruction Independent Component Analysis (RICA) 
unsupervised features extraction technique at each layer to classify depth images with their grayscale $2 \mathrm{D}$ image.

Basically, on each layer, random patches of the grayscale and depth images are used to learn the $R^{2} I C A$ filters. Then, the filters are applied at the whole grayscale and depth images and pooled using spatial pooling and local contrast normalization. The resulting maps are used as input for the next layer.

\subsection{Fuss Convolutional Neural Networks}

Introduced by Eitel et al. (2015), Fuss-CNN method consists of a deep learning architecture of two separate convolutional neural networks: one for the RGB input and other to the depth map. Both are combined with a fusion network. The network was trained in three separate training stages: (i) train the first network with RGB data; (ii) the second with depth images; and (iii) train both networks together with the fusion network.

\subsection{Chapter Considerations}

From handcrafted to sophisticated machine learning techniques, the presented methods extract shape features using different approaches and different 3D images representations. Also, the presented methods participated in the planning of the proposed methods.

For instance, the transform-based methods (trace, Radon and Radial Integration) provided theoretical basis which inspired the RPHSD method transformation from a 3D to 1D structures. Also, the PFH and FPFH inspired the use of histograms as these 1D structures. On the other hand, the VoxNet conversion from shape to occupancy grid was a good start point when the problem to work with shapes using co-occurrence matrices arose for the COMSD.

Further, CFK, $R^{2} I C A$ and FussCNN are the methods with highest accuracy of the University of Washington RGBD dataset, which was used for the proposed methods' validation and comparison.

Finally, this chapter presented methods on 3D shapes classification which are related to the methods proposed in this dissertation. 


\section{CO-OCCURRENCE MATRICES SHAPE DESCRIPTOR 3D}

Co-Occurrence Matrices (COM), also known as Spatial Dependency Matrices (SDM), are widely used to extract features from $2 \mathrm{D}$ and $3 \mathrm{D}$ textures. These features rely on the color transitions of the image. In this chapter are presented previous works on co-occurrence matrices, the method steps to extract them from a colourized 2D and 3D images, the features used in this project and a adaptation for 3D shapes: the Co-Occurrence Matrices Shape Descriptor (COMSD).

\subsection{Previous Works}

Regarding 2D images, Haralick and Shanmugam (1973) firstly presented features extraction from COM computed of 2D images for textures classification. Basically, this method computes statistical properties from the spatial relationships of grayscale values on the image.

Also, despite being introduced in 1973, there are still recent researches in computer vision with COM, for instance:

- In the paper from (OTHMEN; SAYADI; FNIAECH, 2013) gray level co-occurrence matrices are used to extract features from 3D textures;

- Sampson et al. (2014) compute COM textural features from pictures of drying apple slices;

- 3D facial expressions were recognized using COM and 3D-LBP in the paper from (YAHIA; SALEM; ABDELKRIM, 2016);

- Lloyd et al. (2017) detected violent and abnormal crowd activity from video frames using COM. 


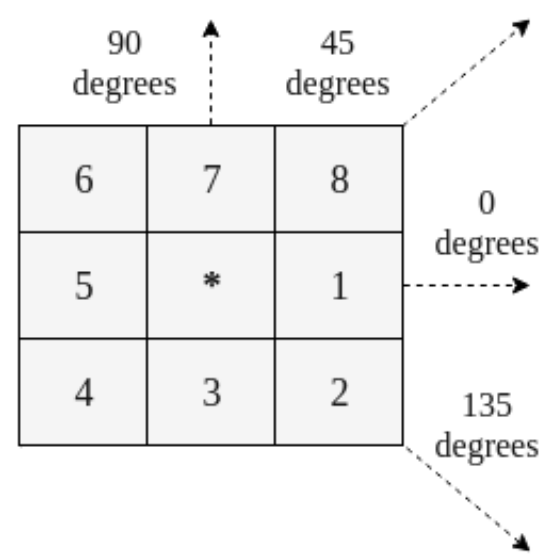

Figure 18 - Possible transition directions for $r=1$.

Finally, the method presented in this chapter was introduced in our recent paper "Cooccurrence matrices for 3D shape classification" (PRZEWODOWSKI FILHO; OSÓRIO, 2017a).

\subsection{Co-Occurrence Matrices}

A COM, initially, stores the number of transitions from a gray level of a point $p_{i}$ to another point with distance $r$ between them and direction $\theta$. For $r=1$, in $2 \mathrm{D}$ images, there are four possible directions of transitions (illustrated in Figure 18) from a pixel to another. For each pixel from the image, its transition is stored in a COM.

For every direction on the image there is a different COM, thus there are four different matrices when $r=1$. It is important to emphasize that a COM of an image with $N$ possible color values will have $N \mathrm{x} N$ dimensions. Figure 19 illustrate an example of quantized 2D image with values that range from 1 to 8 and its horizontal co-occurrence matrix.

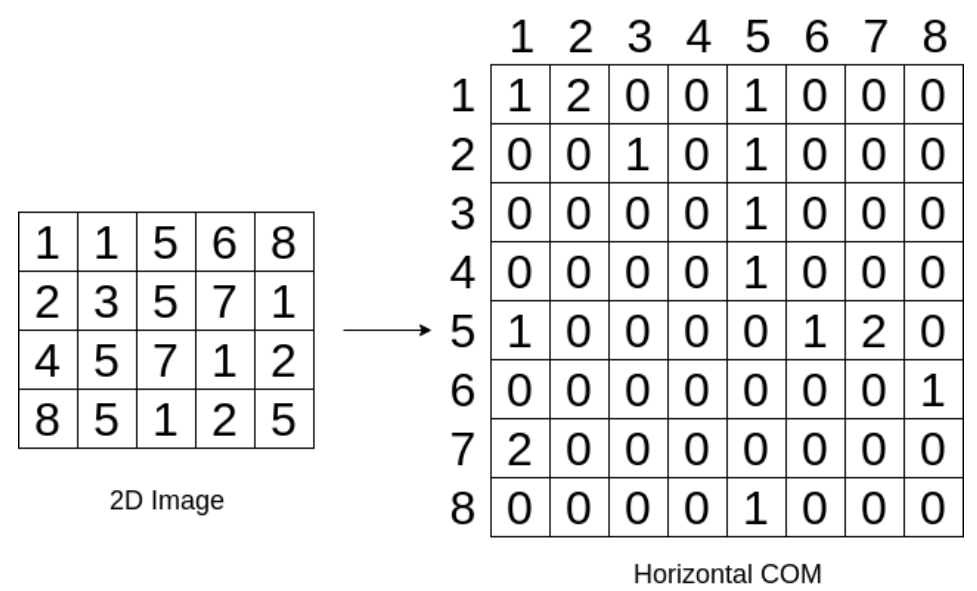

Figure 19 - Example of a horizontal co-occurrence matrix for a 2D image. Notice that there are two transitions from value 1 for 2 in the image and the COM at row 1 and column 2 has value 2. On the other hand, there are no transitions from 4 to 3 , so the COM value at row 4 and column 3 is 0 . 


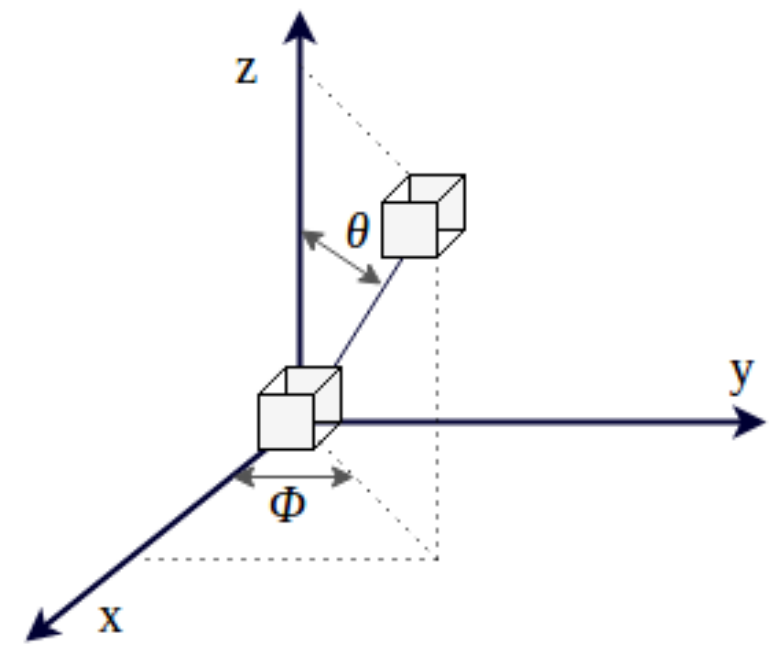

Figure 20 - Angles on 3D images.

\begin{tabular}{c|c} 
Direction Number & Degree Direction $(\theta, \phi)$ \\
\hline 1 & $\left(0^{\circ}, 0^{\circ}\right)$ \\
2 & $\left(45^{\circ}, 0^{\circ}\right)$ \\
3 & $\left(90^{\circ}, 0^{\circ}\right)$ \\
4 & $\left(135^{\circ}, 0^{\circ}\right)$ \\
5 & $\left(0^{\circ}, 45^{\circ}\right)$ \\
6 & $\left(0^{\circ}, 90^{\circ}\right)$ \\
7 & $\left(0^{\circ}, 135^{\circ}\right)$ \\
8 & $\left(90^{\circ}, 45^{\circ}\right)$ \\
9 & $\left(90^{\circ}, 135^{\circ}\right)$ \\
10 & $\left(45^{\circ}, 45^{\circ}\right)$ \\
11 & $\left(45^{\circ}, 135^{\circ}\right)$ \\
12 & $\left(135^{\circ}, 45^{\circ}\right)$ \\
13 & $\left(135^{\circ}, 135^{\circ}\right)$
\end{tabular}

Table 1 - Table of possible directions for transitions between voxels. Source: (OTHMEN; SAYADI; FNIAECH, 2013).

On 3D images, another angle must be considered (3D spherical coordinates, considering $\theta$ and $\phi$ ), resulting on thirteen possible directions for the voxel grid intensity transition analysis (KURANI et al., 2004; OTHMEN; SAYADI; FNIAECH, 2013; MAHMOUD-GHONEIM et al., 2003), as shown in Figure 20 and on Table 1.

After computing all the transitions, the COM is normalized dividing all elements by the total number of transitions, yielding stochastic matrices, where each cell is the probability of a transition to happen. 


\begin{tabular}{c|c} 
Feature & Formula \\
\hline Entropy & $-\sum_{r}^{M} \sum_{c}^{M} P[r, c] \log (P[r, c])$ \\
Energy & $\sum_{r}^{M} \sum_{c}^{M}(P[r, c])^{2}$ \\
Contrast & $\sum_{r}^{M} \sum_{c}^{M}(r-c)^{2} P[r, c]$ \\
Homogeneity & $\sum_{r}^{M} \sum_{c}^{M} \frac{P[r, c]}{1+|r-c|}$ \\
Sum mean $(\mu)$ & $\frac{1}{2} \sum_{r}^{M} \sum_{c}^{M}(r P[r, c]+c P[r, c])$ \\
Variance $\left(\sigma^{2}\right)$ & $\frac{1}{2} \sum_{r}^{M} \sum_{c}^{M}\left((r-\mu)^{2} P[r, c]+(c-\mu)^{2} P[r, c]\right)$ \\
Correlation & $\sum_{r}^{M} \sum_{c}^{M} \frac{(r-\mu)(c-\mu) P[r, c]}{\sigma^{2}}$
\end{tabular}

Table 2 - Each statistical feature associated with its formula. $M$ is the size of the COM $P$ and $P[r, c]$ is the value from $P$ at the row $r$ and column $c$.

\subsection{Features Extraction}

After computing all the normalized COM, many statistical features can be computed from them. Kurani (KURANI et al., 2004) provides a table with each feature description and its formula. In this work, the following features have been computed: entropy, energy, contrast, homogeneity, sum mean, variance and correlation. Each formula is represented on the Table 2. In the paper of Haralick and Shanmugam (1973) more features and their intuitive meaning are presented.

These statistical features represent the distribution of the transitions for every direction.

\subsection{Shape Descriptor}

So far, the extracted features describe texture from images with color information. However, the goal is shape description, so an adaptation is necessary.

Considering a shape as the input for the algorithm, it has to be converted to an image representation that is able to provide value transition between the point neighbors, as voxel grids. Furthermore, color information must be discarded, as the goal is shape description. Thus, the voxel grid must be binary, in which a voxel has value 1 if is filled and 0 if is empty. This means that each COM has dimensions $2 \times 2$ corresponding to:

- Cell $(0,0)$ : the number of transitions from an empty voxel to another empty voxel;

- Cell $(0,1)$ : the number of transitions from an empty voxel to a filled voxel;

- Cell (1,0): the number of transitions from a filled voxel to an empty voxel;

- Cell $(1,1)$ : the number of transitions from a filled voxel to another filled voxel.

Finally, the features can be computed from the obtained COM. 
Summarizing, the COMSD steps are: (i) given a 3D shape as input, the COMSD converts it to a binary voxel grid representation; (ii) with the voxel grid, a COM is computed for each of the 13 possible directions; (iii) from each COM, extract the selected features.

In the first step, the algorithm iterates over each point of the point cloud to compute the voxel grid, then its complexity is $O(n)$, where $n$ is the size of the point cloud. In the second step, the algorithm iterates over the voxel grid, which has $O(k \cdot l \cdot m)$ complexity, where $k, l$ and $m$ are the number of rows, columns =- and depth values the voxel grid has. For the third step, since a $3 \mathrm{D}$ shape COM has constant dimensions $2 \times 2$, the feature extraction step has $O(1)$ complexity. Finally, the complexity of the algorithm is $O(n+k \cdot l \cdot m)$. 

CHAPTER

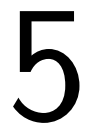

\section{D COMPLEX NETWORK SHAPE DESCRIPTOR}

Complex networks are well known from their use in the analysis of dissemination of diseases and spread of dissemination. In this chapter are presented previous applications in computer vision, how to represent a 3D point cloud as a complex network, and how to extract features from them.

\subsection{Related Works}

Complex network approaches in computer vision started when Backes, Casanova and Bruno (2009) proposed a method based on complex networks that computes features for boundary shape analysis on 2D images. After that, much research on 2D images textures with complex networks has been done. Some of them:

- The method proposed by Backes, Martinez and Bruno (2011) generates graphs from the self-avoiding walks algorithm ${ }^{1}$, from which features are extracted to describe the image texture;

- Tang et al. (2012) proposed an application of the complex networks for 3D objects recognition, but the graphs were extracted from $2 \mathrm{D}$ views of the objects;

- In Backes, Casanova and Bruno (2013), each pixel from the image is considered a vertex from the network and its connection weight with other pixels is proportional to the distance of the vertices and the difference of their intensities;

1 An algorithm that, given a point from a graph, finds a path in which all points can be visited without repetition. 

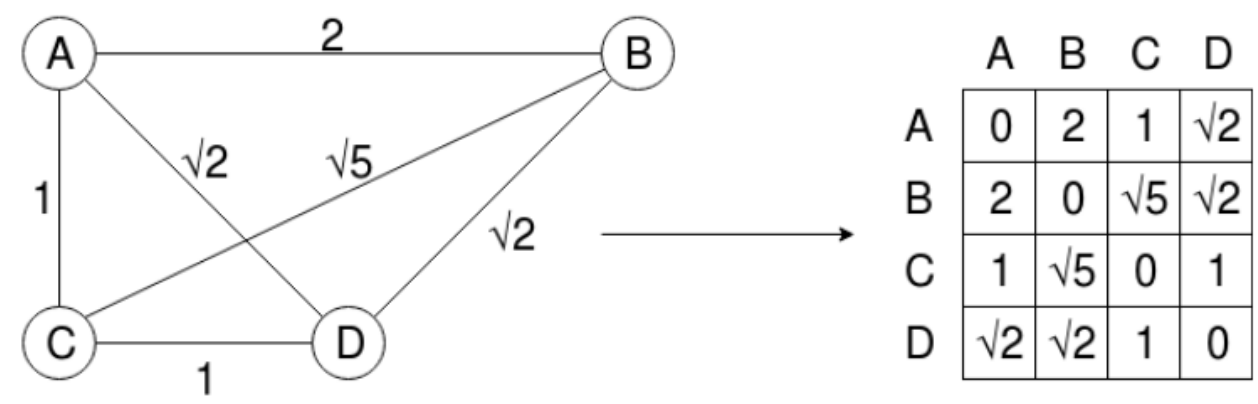

Figure 21 - Example of an undirected graph (left) and its corresponding adjacency matrix (right).

- Gonçalves and Bruno (2013), presented an application of the self-avoiding walks graphs approach on dynamic textures for classification, segmentation and clustering that were better than the state-of-the-art methods on dynamic textures;

- Florindo, Landini and Bruno (2016) proposed an application of the complex networks for $2 \mathrm{D}$ images texture recognition that uses the pixel intensity as a third dimension of the pixel position.

In this chapter, the method proposed was presented in our recent paper "Complex network shape descriptor for 3D objects classification" (PRZEWODOWSKI FILHO; OSÓRIO, 2017b).

\subsection{Complex Networks Representation}

Basically, a complex network works with concepts of graph theory and statistical mechanics (BACKES; CASANOVA; BRUNO, 2009). It is composed by nodes (or vertices) and edges, which are undirected. Each node can or can not be connected with other vertices. The degree of a node is the number of nodes that are directly connected to it.

Then, given a list of points of size $N$, it can be represented by an undirected adjacency matrix $P$ in which each weight $P_{i, j}$ is

$$
P_{i, j}=d(i, j)
$$

where $i$ and $j$ are the indexes associated to two different points of the image and $d$ computes the distance between $i$ and $j$. Figure 21 illustrate an example of an undirected graph and its adjacency matrix.

In previous works, these points belonged to a 2D image and either were 2D (their position in the image) or 3D, considering the gray scale level as a third dimension. The method presented in this chapter supports spatial 3D points (without color information).

Since $3 \mathrm{D}$ point clouds are a list of points, it is plausible to assume that each point is a vertex from the network. Thus, the distance function $d($.$) for the adjacency matrix representation$ 
can be computed by the Euclidean distance, as follows:

$$
d(i, j)=\sqrt{\left(i_{x}-j_{x}\right)^{2}+\left(i_{y}-j_{y}\right)^{2}+\left(i_{z}-j_{z}\right)^{2}} .
$$

\subsection{Feature Extraction}

After computing all the weights, $P$ is normalized by its maximum weight $P_{\max }$

$$
P_{i, j} \leftarrow \frac{P_{i, j}}{P_{\max }}
$$

The edge cutting phase applies a threshold $T$ on the original network and generates a network $G$ with weights

$$
G_{i, j}= \begin{cases}1 & P_{i, j} \leq T \\ 0 & P_{i, j}>T\end{cases}
$$

which removes distant vertices connections.

According to Backes, Casanova and Bruno (2009), the edge cutting phase represents the evolution of the network. This phase is repeated from $T_{\text {start }} \geq 0$ to $T_{\text {end }} \leq 1$ and $T_{\text {end }}>T_{\text {start }}$ with a step $\Delta T$. On each step, the degree from all vertices of $G$ are computed. Furthermore, those degrees are normalized by $N$, resulting on $D$, and the features maximum degree $D_{\max }(T)$ and average degree $D_{\text {avg }}(T)$ are computed.

Thus, the descriptor $\phi$ is given by the set of all $D_{\max }\left(T_{k}\right)$ and $D_{\text {avg }}\left(T_{k}\right)$ computed:

$$
\begin{array}{r}
\phi=\left\{D_{\text {avg }}\left(T_{\text {start }}\right), D_{\text {max }}\left(T_{\text {start }}\right), D_{\text {avg }}\left(T_{1}\right), D_{\text {max }}\left(T_{1}\right),\right. \\
\left.D_{\text {avg }}\left(T_{2}\right), D_{\text {max }}\left(T_{2}\right), \cdots, D_{\text {avg }}\left(T_{\text {end }}\right), D_{\text {max }}\left(T_{\text {end }}\right)\right\}
\end{array}
$$

for each $T_{k}$ in $T_{\text {start }} \leq T_{k} \leq T_{\text {end }}$ and $T_{k}=T_{k-1}+\Delta T$.

Figure 22 illustrates this process which, depending on the edge cutting threshold, and the maximum and average distance obtained between the points, may result in a spatial partition of the graph.
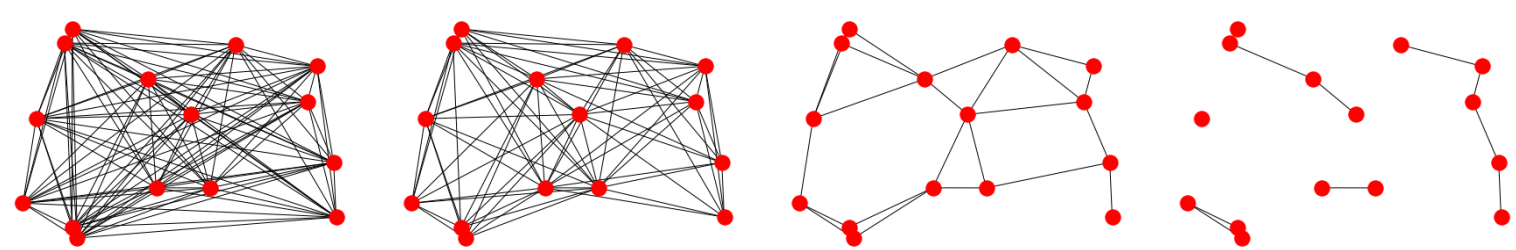

Figure 22 - Example of connected edges with different $T$. From the left to the right: Original network; edges when $T=0.8$; edges when $T=0.4$; edges when $T=0.3$.

For each edge cutting step, the algorithm iterates over the adjacency matrix. Consider that the adjacency matrix size is $N^{2}$ and that the number of times the edge cutting step is executed is $S$, the algorithm's complexity is $O\left(S \cdot N^{2}\right)$. In the usual case, since $N^{2} \gg S$, the complexity is $O\left(N^{2}\right)$. 
Finally, the set of values (maximum and average degrees) obtained from this process is then adopted as the descriptor $\phi$ of the shape. The descriptors from all shapes of the dataset are then provided to a machine learning algorithm in order to learn how to separate and classify them. 
CHAPTER

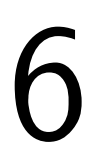

\section{REFERENCE POINTS HISTOGRAMS SHAPE DESCRIPTOR}

As seen on the previous chapters, distance is a popular measurement for shape features extraction. For instance:

- The NARF method (more at Chapter 3) uses a weighted distance function on an interest point's neighborhood to compute its descriptor;

- The MPPF method (more at Chapter 3) uses the metric distance between the center of the viewpoint and each geometric edge point computed.

- The CNSD method (more at Chapter 5) uses distance to determine edge cuts in a complex network computed from a point cloud. From these cuts, features are extracted from the generated graph.

In this chapter, it is presented the Reference Points Shape Descriptor (RPHSD), which computes histograms of distances for a given set of reference points and a point cloud. These histograms features provide statistical features that describe the shape.

Also, heuristics for parameters choice, extractable features and algorithm analysis are provided in the following sections.

\subsection{Algorithm Foundations}

When designing the RPHSD, our first intent was to map the points from 3D to $1 \mathrm{D}$, but it can also be used to map from $2 \mathrm{D}$ points to $1 \mathrm{D}$. For that, a possibility is to compute a histogram of distances $d_{n}(P, Q)$ from a reference point $Q$ to all the cloud points in which the index for each computed distance $i($.$) is$

$$
i(P, Q)=M \cdot\left(d_{n}(P, Q)\right)
$$



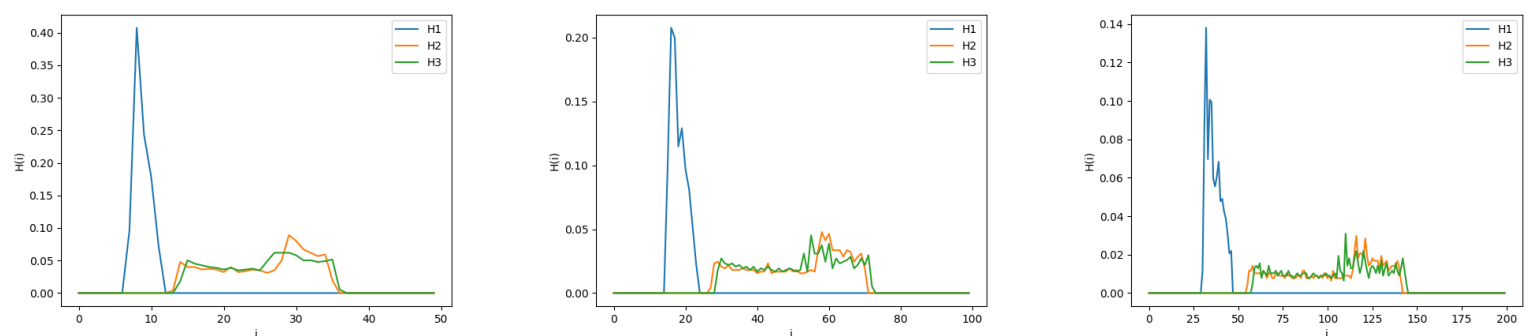

Figure 23 - Computed histograms for a box. From the left to the right: $M=50 ; M=100$; and $M=200$.

where $P$ is a point from the point cloud; $M$ is the size of the histogram; and $d_{n}(P, Q)$ is a normalized distance function (values between 0 and 1 ). The normalized distance can be achieved dividing the distance function $d($.$) by the maximum possible distance of the system d_{\text {max }}$,

$$
d_{n}(P, Q)=\frac{d(P, Q)}{d_{\max }}
$$

E.g., on a 2D signal $f(x, y)$, with $x, y \in \mathbb{R} \mid-1 \leq x, y \leq 1$, the maximum distance can be given by the distance between $A(-1,-1)$ and $B(1,1)$, which is $d_{\max }=2 \sqrt{2}$.

The index returned by the index function $t=i($.$) is used to increment a histogram of$ points distribution on different distances in respect to the reference point. For a higher $M$, less likely two points will provide the same index, but the histograms may be more susceptible to noise. On the other hand, when $M$ has lower values, the sensibility (the capacity for detecting details) of the method is reduced.

Still, these histograms can map two different points with the same distance to the same position, since points can be anywhere around the reference point. For instance, consider $Q$ a reference point at $(0,0,0) ; P_{1}=(1,0,0)$ and $P_{2}(0,1,0)$ two points from a point cloud. $P_{1}$ and $P_{2}$ have the same Euclidean distance to $Q$. So, how to solve this ambiguity problem?

\subsection{Reference Points Selection}

One way to map the points to the reference with less (or any) redundancy is to have many different reference points, like in Global Positioning System (GPS) devices.

On 2D images, with 3 reference points it is possible to map the points without ambiguity. In Figure 23, the histogram $H(x)$ were computed using three reference points. Notice that, even with variable histogram size $M$, these histograms have very similar shape for a given image. Also, notice also that, for higher $M$, the shape of the histogram is more sensitive to the details of the object shape.

Also different histogram shapes can be observed from different objects. For instance, in Figure 24, three histograms were computed using the same reference points for three different objects: a box, a comb and a banana. 

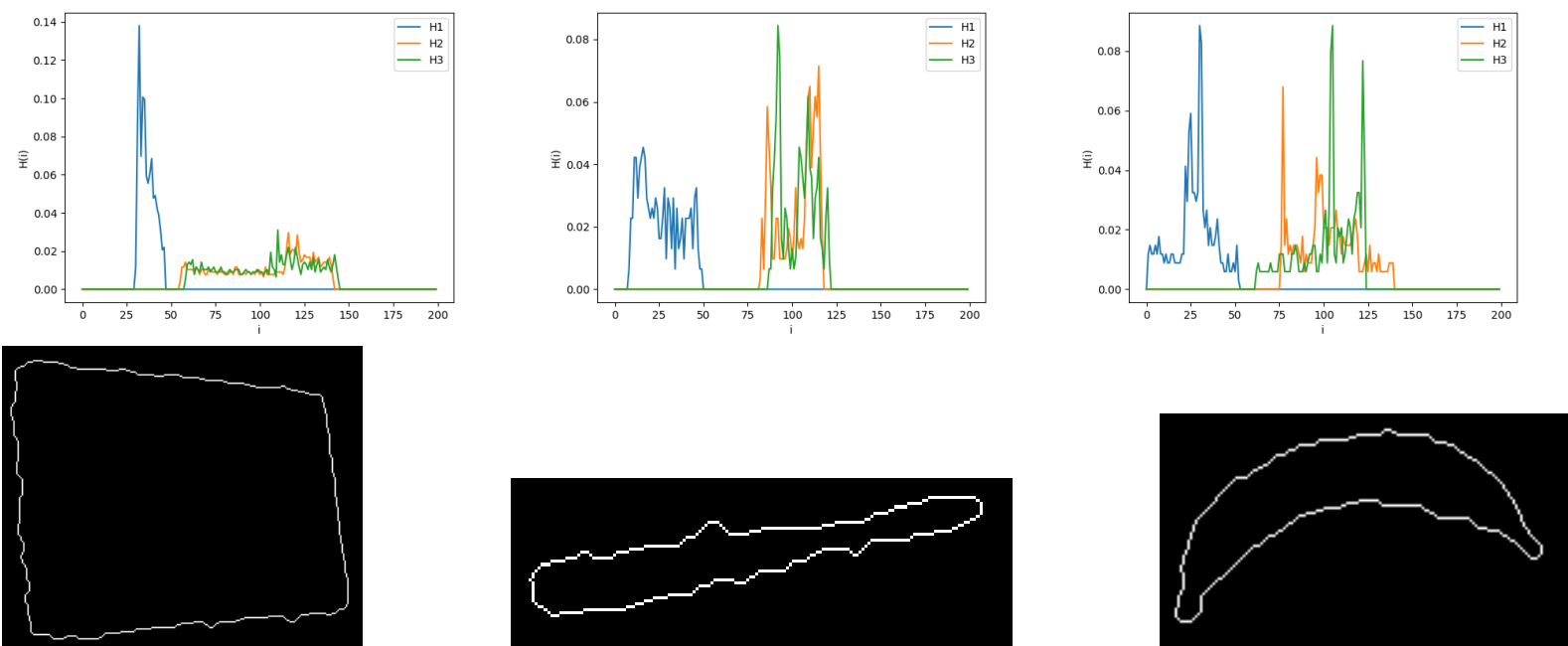

Figure 24 - On the top, three different histograms triples $(M=200)$ from: a box (left); a comb (middle); and a banana (right). On the bottom, the shapes from: a box (left); a comb (middle); and a banana (right).

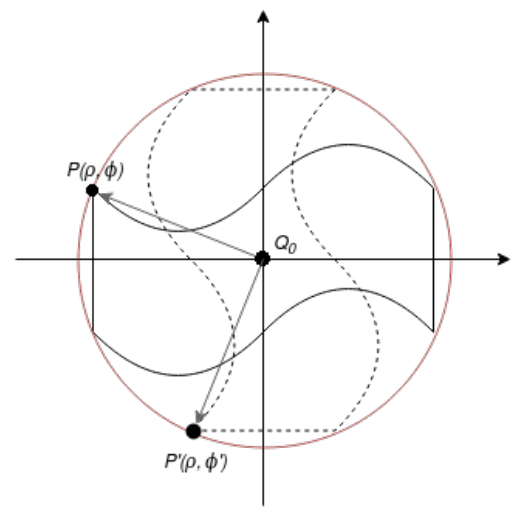

Figure 25 - The invariance against rotation on the center of mass (points represented on a polar coordinate system). Notice that, for any direction that the shape rotates, the points will remain at the same distance $\rho$ from the center.

Still, there are translation, scaling and rotation issues to be dealt with. The translation and scaling issues can be solved by applying max-min normalization on the cloud and transposing it to its mass center. The rotation invariance issue is a more complex problem that is explored along this section.

Given that the shape is scaled and translated to its mass center, one recommended reference point is the origin, since any rotation on the cloud still gives the same distance from a point to the origin (as can be seen in Figure 25).

However, there are other reference points that must be taken in account. One approach to select rotation invariant reference points would be detecting and using image key points as reference points. This approach may find rotation invariant points on the cloud, but the computational time to process the image and/or to compute the points may be high for larger clouds. Also, a different number of points at different positions may be found for slightly different 


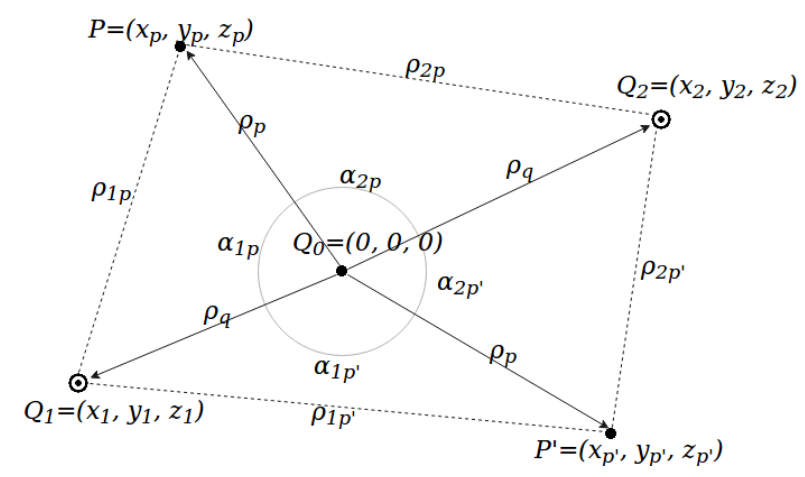

Figure 26 - The points in the 3-dimensional space.

clouds, leading to a difference at the number of histograms. This last problem can be dealt the same way the fixed reference points deal with the rotation variance, which is going to be seen later at this section.

On the other hand, to compute a fixed number of points that will not change their position tend to be a faster approach and can also lead to rotation invariant results.

Consider two reference points $Q_{1}=\left(x_{1}, y_{1}, z_{1}\right)$ and $Q_{2}=\left(x_{2}, y_{2}, z_{2}\right)$ with the same distance to the origin. Also, consider a point from the cloud $P=\left(x_{p}, y_{p}, z_{p}\right)$ and its rotated position $P^{\prime}=\left(x_{p^{\prime}}, y_{p^{\prime}}, z_{p^{\prime}}\right)$, respectively (illustrated in Figure 26). Furthermore, let $\rho_{1 p}, \rho_{2 p}, \rho_{1 p^{\prime}}$ and $\rho_{2 p^{\prime}}$ be the distance between $Q_{1}$ and $P, Q_{2}$ and $P, Q_{1}$ and $P^{\prime}$, and $Q_{2}$ and $P^{\prime}$, respectively. Besides, $\alpha_{1 p}, \alpha_{1 p^{\prime}}, \alpha_{2 p}$ and $\alpha_{1 p}$ are the angles, respectively, $\angle Q_{1} Q_{0} P, \angle Q_{1} Q_{0} P^{\prime}, \angle Q_{2} Q_{0} P$ and $\angle Q_{2} Q_{0} P^{\prime}$.

For the histograms of $Q_{1}$ and $Q_{2}$ be the same after the rotation, either: (a) the distance between $Q_{1}$ and $P$ is the same distance between $Q_{2}$ and $P^{\prime}\left(\rho_{1 p}=\rho_{2 p^{\prime}}\right)$; or (b), the distance between $Q_{1}$ and $P$ is the same distance between $Q_{1}$ and $P^{\prime}\left(\rho_{1 p}=\rho_{1 p^{\prime}}\right)$.

We have that

$$
\begin{array}{r}
\rho_{1 p^{\prime}}^{2}=\overbrace{x_{1}^{2}+y_{1}^{2}+z_{1}^{2}}^{\rho_{q}^{2}}-2\left[x_{1} x_{p^{\prime}}+y_{1} y_{p^{\prime}}+z_{1} z_{p^{\prime}}\right]+\overbrace{x_{p^{\prime}}^{2}+y_{p^{\prime}}^{2}+z_{p^{\prime}}^{2}}^{\rho_{p}^{2}} \\
\rho_{2 p}^{2}=\overbrace{x_{2}^{2}+y_{2}^{2}+z_{2}^{2}}^{\rho_{q}^{2}}-2\left[x_{2} x_{p}+y_{2} y_{p}+z_{2} z_{p}\right]+\overbrace{x_{p}^{2}+y_{p}^{2}+z_{p}^{2}}^{\rho_{p}^{2}} \\
\rho_{1 p}^{2}=\overbrace{x_{1}^{2}+y_{1}^{2}+z_{1}^{2}}^{\rho_{q}^{2}}-2\left[x_{1} x_{p}+y_{1} y_{p}+z_{1} z_{p}\right]+\overbrace{x_{p}^{2}+y_{p}^{2}+z_{p}^{2}}^{\rho_{p}^{2}} \\
\rho_{2 p^{\prime}}^{2}=\overbrace{x_{2}^{2}+y_{2}^{2}+z_{2}^{2}}^{\rho_{q}^{2}}-2\left[x_{2} x_{p^{\prime}}+y_{2} y_{p^{\prime}}+z_{2} z_{p^{\prime}}\right]+\overbrace{x_{p^{\prime}}^{2}+y_{p^{\prime}}^{2}+z_{p^{\prime}}^{2}}^{\rho_{p}^{2}}
\end{array}
$$

From (a),

$$
\rho_{1 p}=\rho_{2 p^{\prime}} \Rightarrow \rho_{1 p}^{2}=\rho_{2 p^{\prime}}^{2}
$$


Which in a few steps, using the equations from (6.3), is

$$
x_{1} x_{p}+y_{1} y_{p}+z_{1} z_{p}=x_{2} x_{p^{\prime}}+y_{1} y_{p^{\prime}}+z_{1} z_{p^{\prime}}
$$

Assuming $\vec{Q}_{1}, \overrightarrow{Q_{2}}, \vec{P}$ and $\vec{P}^{\prime}$ the vectors from the origin to the points $Q_{1}, Q_{2}, P$ and $P^{\prime}$ respectively, we have - from the analytical geometry - two inner products in (6.4), leading to

$$
\vec{Q}_{1} \cdot \vec{P}=\vec{Q}_{2} \cdot \vec{P}^{\prime}
$$

Applying the same logic to (b), we have

$$
\vec{Q}_{1} \cdot \vec{P}=\vec{Q}_{1} \cdot \vec{P}^{\prime}
$$

Thus, the reference points capture invariant shapes when one of the conditions (6.5) or (6.6) is filled. In respect to rotation angles, what does this mean?

From the analytical geometry, we also have that

$$
\cos (\alpha)=\frac{\vec{u} \cdot \vec{v}}{\|\vec{u}\| \cdot\|\vec{v}\|}
$$

Applying (6.7) in (6.5), we have that

$$
\cos \left(\alpha_{1 p}\right)=\cos \left(\alpha_{2 p^{\prime}}\right)
$$

leading to the condition

$$
\left|\alpha_{1 p}\right|=\left|\alpha_{2 p^{\prime}}\right|
$$

Or, from (6.7) in (6.6),

$$
\left|\alpha_{1 p}\right|=\left|\alpha_{1 p^{\prime}}\right|
$$

What conclusions can we observe from these equations? For instance, consider that the points belong to a two dimensional system and $\theta_{1}, \theta_{2}, \theta_{p}$ and $\theta_{p^{\prime}}$ are the angles from $Q_{1}, Q_{2}, P$ and $P^{\prime}$ in respect to the horizontal axis. Also, $\alpha_{1 p}=\theta_{p}-\theta_{1}$ and $\alpha_{2 p^{\prime}}=\theta_{p^{\prime}}-\theta_{2}$. Thus, from the condition (6.9), if $\theta_{p} \geq \theta_{1}$ and $\theta_{p^{\prime}} \geq \theta_{2}$, or $\theta_{p}<\theta_{1}$ and $\theta_{p^{\prime}}<\theta_{2}$, then we have:

$$
\theta_{p}-\theta_{1}=\theta_{p^{\prime}}-\theta_{2} \Leftrightarrow \theta_{p^{\prime}}=\theta_{p}+\left(\theta_{2}-\theta_{1}\right)
$$

From that, one situation can be noticed: if $\theta_{p}$ is between $\theta_{1}$ and $\theta_{2}$, and if the cloud rotates $\left|\theta_{2}-\theta_{1}\right|$ around its mass center, then the conditions of invariance would be satisfied. This conclusion gives us a notion of resolution of invariance for the method.

For instance, let $Q_{1}=(-1,0), Q_{2}=(1,0)$ (the angle between them is $180^{\circ}$ ) and $P=$ $(0.8660,0.5000)\left(30^{\circ}\right.$ from $\left.Q_{1}\right)$, illustrated in Figure 27 . The points that would satisfy the 

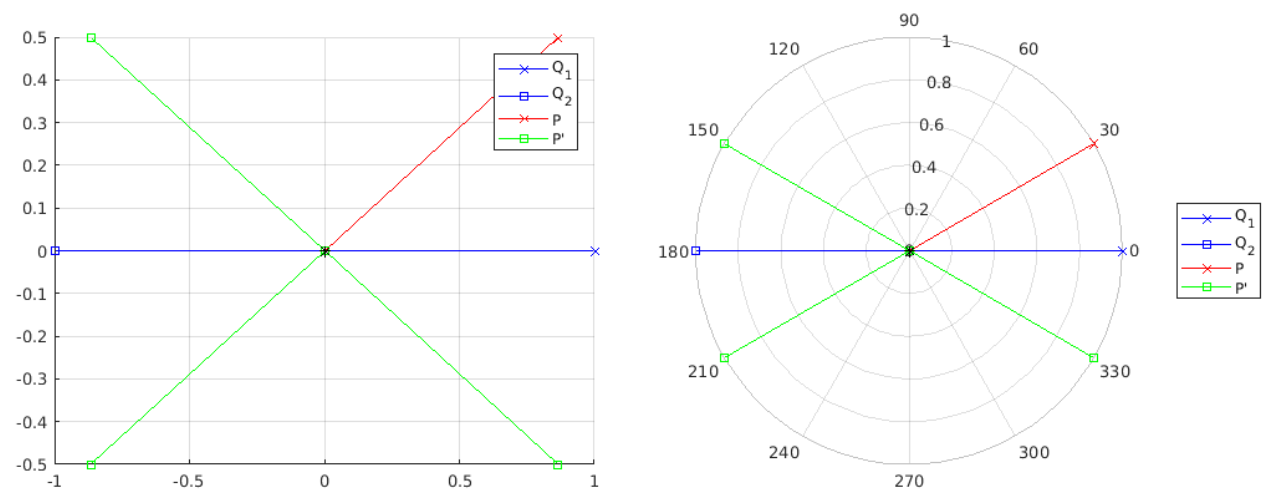

Figure 27 - Points from the example in: (left) Cartesian coordinates; (right) polar coordinates. The blue dots are the reference points, the red dot is $P$ and the green dots are the possible $P^{\prime}$.

conditions (without the trivial case, where $\left.P^{\prime}=P\right)$ are: $P^{\prime}=(0.8660,-0.5000)\left(-30^{\circ}\right.$ from $\left.Q_{1}\right)$, $P^{\prime}=(-0.8660,-0.5000)\left(30^{\circ}\right.$ from $\left.Q_{2}\right)$ and $P^{\prime}=(-0.8660,0.5000)\left(-30^{\circ}\right.$ from $\left.Q_{2}\right)$.

As said before, if $\theta_{p}$ is between $\theta_{1}$ and $\theta_{2}$ and the cloud rotates $\left|\theta_{1}-\theta_{2}\right|$ around its mass center, then the invariance against rotation would be assured. However, how to assure that $\theta_{p}$ is between $\theta_{1}$ and $\theta_{2}$ ? A way to assure this condition is to fill the circumference with $N$ reference points that have the same angular distance $\Delta \alpha$ with the rule:

$$
N=\frac{360}{\Delta \alpha} \text {. }
$$

For instance, if one wants to the algorithm be invariant to rotations of at least $45^{\circ}$, it should use $N=8$ reference points with angular distance of $45^{\circ}$ from each other (Figure 28).

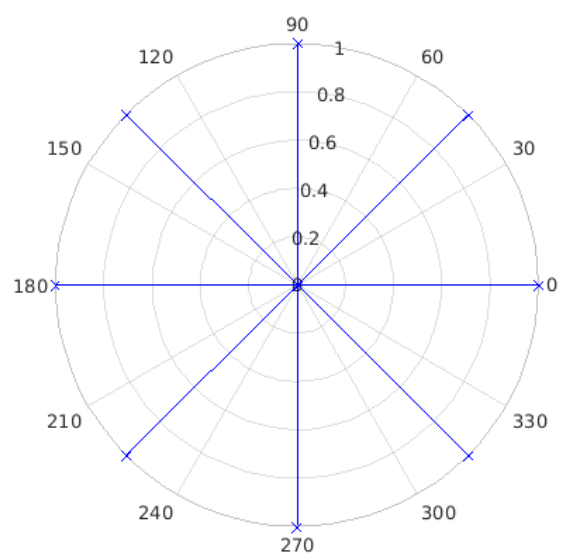

Figure 28 - The eight reference points with angular distance $\Delta \alpha=45^{\circ}$, computed using the Equation (6.11).

In Figure 29, the shape of a hand towel was rotated four times, $90^{\circ}$ each time. Using four reference points in positions $Q_{1}=(1,0), Q_{2}=(0,1), Q_{3}=(-1,0)$ and $Q_{4}=(0,-1)$, the produced incremental histograms were illustrated on Figure 30.

It is important to emphasize that the histograms from the different reference points after the rotations are only going to stay the same if the condition (6.10) is filled. Else, if the condition 

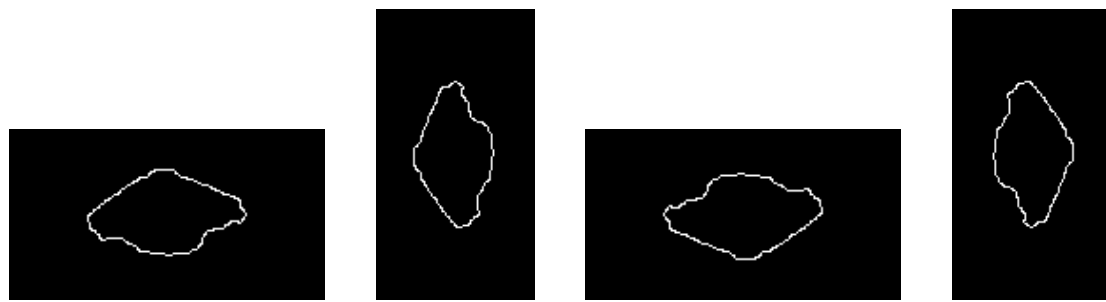

Figure 29 - Shapes of a hand towel. From the left to the right: without rotation; rotated 90 degrees; rotated 180 degrees; rotated 270 degrees.
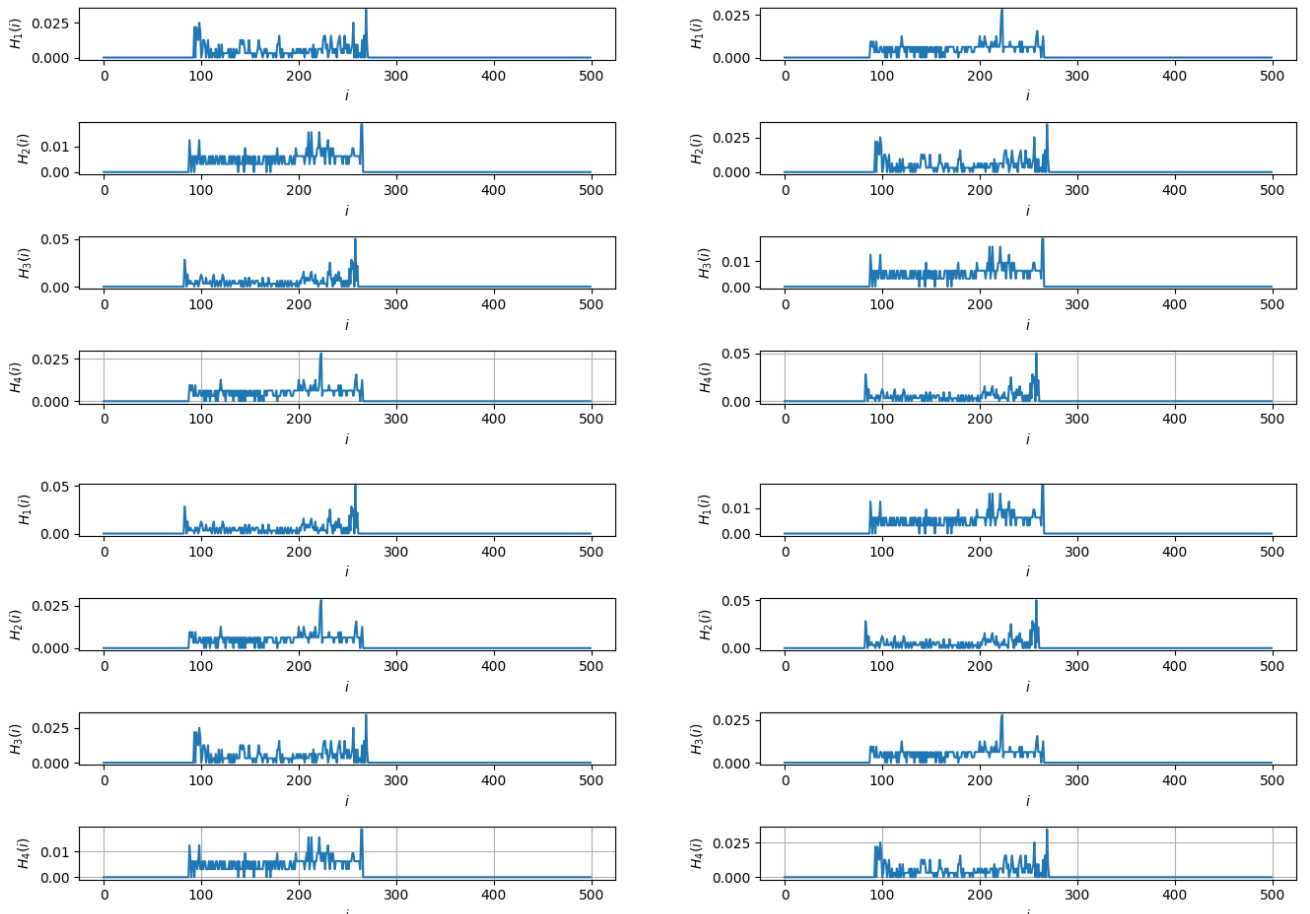

Figure 30 - The histograms from the rotated shapes from the shape: (i) without rotation (top left); (ii) with 90 degrees rotation (top right); (iii) with 180 degrees rotation (bottom left); (iv) with 270 degrees rotation (bottom right). Notice that the histograms are the same, but swapped. For instance: $H_{1}$ from (i) is the same as $H_{2}$ from (ii), which is the same as $H_{3}$ from (iii) and $H_{4}$ from (iv).

(6.9) is filled, then the histograms will be swapped. One way to avoid this situation compute the average and the standard deviation from each feature of the histograms.

In 3D images, to find equally spaced points over a sphere is a issue known as the spherical covering problem. Several algorithms exist, but they have limitations, such as imprecision or heterogeneous spaces. Two of those algorithms are the Golden Spiral Method and the Fibonacci Sphere Algorithm. Other solutions can be employed, like selecting only points that belong to the planes $X Y, X Z$ and $Y Z$, or evenly distributing them in circumferences on different longitudes, but then the conditions imposed in this section are not assured. 


\subsection{Features}

The features are extracted from each histogram. Three possible features are: (i) variance, (ii) entropy and (iii) inverse difference moment. But, since the histograms can be swapped, using these values for each histogram will provide a variant-to-rotation descriptor. A way around this situation is to compute the average and the standard deviation for each feature of the histograms.

Assuming that $N$ is the number of reference points, suppose that the set of: variances is $v=\left\{v_{1}, v_{2}, \ldots, v_{N}\right\}$; entropies is $\varepsilon=\left\{\varepsilon_{1}, \varepsilon_{2}, \ldots, \varepsilon_{N}\right\}$; and of inverse difference moments is $\tau=$ $\left\{\tau_{1}, \tau_{2}, \ldots, \tau_{N}\right\}$. Also, assume that the set of averages and the standard deviations of the features variance, entropy and inverse difference moment are $\mu=\left\{\mu_{v}, \mu_{\varepsilon}, \mu_{\tau}\right\}$ and $\sigma=\left\{\sigma_{v}, \sigma_{\varepsilon}, \sigma_{\tau}\right\}$. If $\mu_{x}$ is the average of a feature $x=v, \varepsilon$ or $\tau$ of a cloud and $\mu_{x^{\prime}}$ is the average of the feature $x^{\prime}=v, \varepsilon$ or $\tau$ of the cloud's rotation (respecting the invariance to rotation condition), and $x$ and $x^{\prime}$ represent the average of the same feature, then:

$$
\mu_{x}=\frac{1}{N} \sum_{i=1}^{N} x_{i}
$$

and

$$
\mu_{x^{\prime}}=\frac{1}{N} \sum_{i=1}^{N} x_{i}^{\prime} .
$$

Since $x$ and $x^{\prime}$ have the same elements but in shifted positions, then one can notice that

$$
\sum_{i=1}^{N} x_{i}^{\prime}=\sum_{i=1}^{N} x_{i}
$$

Thus $\mu_{x}=\mu_{x^{\prime}}$.

For $\sigma_{x}$ and $\sigma_{x^{\prime}}$, we have

$$
\sigma_{x}=\sqrt{\frac{1}{N} \sum_{i=1}^{N}\left(x_{i}-\mu_{x}\right)^{2}}
$$

and

$$
\sigma_{x^{\prime}}=\sqrt{\frac{1}{N} \sum_{i=1}^{N}\left(x_{i}^{\prime}-\mu_{x^{\prime}}\right)^{2}} .
$$

We have computed before that $\mu_{x}=\mu_{x^{\prime}}$. Also, we know that $x$ and $x^{\prime}$ have the same elements in shifted positions. Then:

$$
\sum_{i=1}^{N}\left(x_{i}-\mu_{x}\right)^{2}=\sum_{i=1}^{N}\left(x_{i}^{\prime}-\mu_{x^{\prime}}\right)^{2} .
$$

Finally, $\sigma_{x}=\sigma_{x^{\prime}}$, assuring that the features are invariant to rotation when the conditions from Section 6.2 are satisfied, what was to be demonstrated. 
With that, the reduced descriptor has a constant size (in the case of this work, the descriptor has size 6), but is smaller if compared to the complete descriptor and can be less discriminative than the complete descriptor is.

\subsection{Algorithm and Analysis}

Summarizing the steps presented on the previous sections:

1. Select a set of reference points;

2. For each reference point, compute the histogram of distances between it and the points from the cloud;

3. For each histogram, compute statistical features and append them to the descriptor;

4. Reduce the descriptor computing the average and the standard deviation for each feature (optional).

These steps are detailed in Algorithm 1. The function normalizedDistance $(p, q)$ computes the distance between the points $p$ and $q$ and divides it by the maximum possible distance. Since points position is normalized between -1 and 1 , the maximum possible distance is between $p=(-1,-1,-1)$ and $q=(1,1,1)$, resulting on $d_{\max }=2 \sqrt{3}$. The function size(.) computes the size of a list. The functions computeAverage(.), computeVariance(.), computeStdDev(.), computeEntropy(.) and computeIDM(.) compute and return, respectively, the average, the variance, the standard deviation, the entropy and the inverse difference moment from an array. The operation $X \leftarrow X \bigcup\left\{x_{a}, x_{b}, \ldots, x_{n}\right\}$ represents the concatenation between the set $\mathrm{X}$ and the values $x_{a}, x_{b}, \ldots, x_{n}$. The input variables for this algorithm are: the normalized point cloud $P$, the set of reference points $Q$ and the size of each histogram $M$, which can also be interpreted as the inverse of the step size. Finally, $H$ is the list of histograms that has $\operatorname{size}(Q)$ histograms.

The reduced descriptor version algorithm is an extension from the Algorithm 1, computing the average and variance from the three lists listVar, listEnt and listIDM, summarized in Algorithm 2.

The first property to be noticed of the RPHSD method is its complexity: it (i) initializes the $K$ histograms ( $K$ is the number of reference points and histograms) with 0 (lines 1 to 4 ), (ii) runs one time at the input cloud to fill $K$ histograms (lines 5 to 10), (iii) normalizes them (lines 11 to 13 ) and (iv) computes their features (lines 18 to 23 ).

Since the steps (i), (iii) and (iv) iterate $K$ times over $M$ sized histograms, they have the complexity $O(K \cdot M)$. The step (ii) has $O(N \cdot K)$ complexity, where $N$ is the size of the cloud. Thus, the algorithm has the complexity $O(K \cdot N+K \cdot M)$. Usually, $K \cdot N$ is orders of greatness bigger than $K \cdot M$ so, in the usual case, the complexity is $O(K \cdot N)$. 

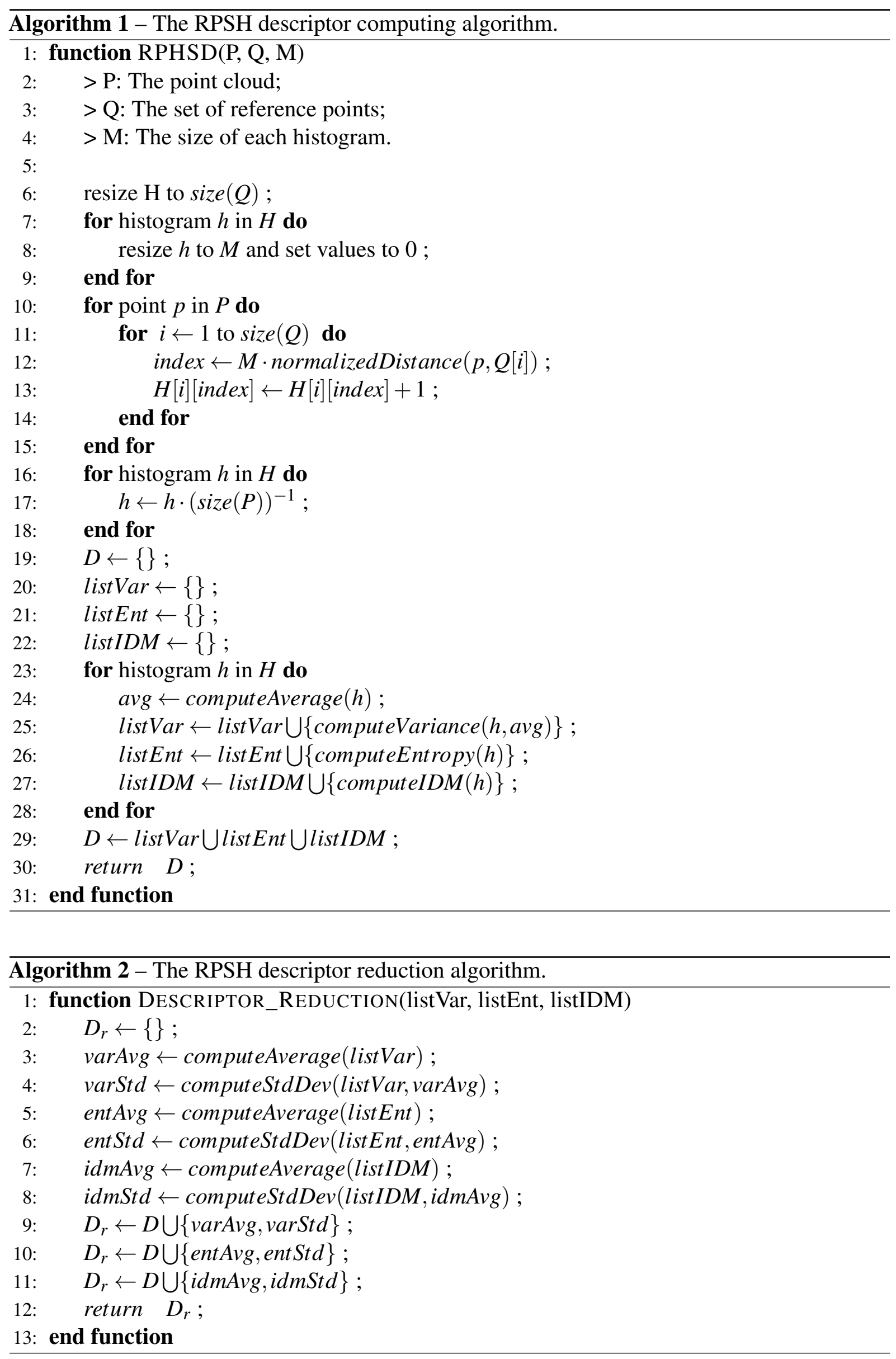
Beyond that, the scale, translation and rotation are assured, as proved on Section 6.2.

Another property is that, since the RPHSD depend only on the histograms and the reference points, it can be applied on input data of any dimension.

The parameters of this algorithm are only the size of the histograms (which can be interpreted as the inverse of the step size of the hypersphere or the sensitivity of the descriptor), the point cloud and the set of reference points. 

CHAPTER

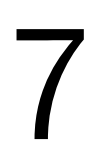

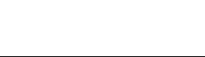

DATASETS

This chapter presents the datasets used to compare this work's results: the University of Washington Dataset (UW-DS), presented in Section 7.1, the ModelNet Dataset (MN-DS), in Section 7.2, and the 3DNet, in Section 7.3; and they are compared in Section 7.4.

\subsection{University of Washington RGB-D Dataset}

The RGB-D dataset, provided by the University of Washington ${ }^{1}$ (LAI et al., 2011), was used in the experiments. Each object instance was obtained from a segmented RGB-D image seen on different poses. Thus, all object instances have some part of them omitted (occlusion), which increases the difficulty of the classification.

This dataset was recorded using a Kinect style 3D camera that records synchronized and aligned 640x480 RGB and depth images (Figure 31). Each object was placed on a turntable and sequences were captured for a whole object rotation, containing around 250 frames per object, which are obtained from 3 different view angles (30, 45 and 60 degrees above the horizon), giving more than 200,000 RGB + Depth frames in the RGB-D object dataset. Also, there are 300 object instances classified into 51 categories, once, for example, the category soda can is divided into physically unique instances like Pepsi Can and Mountain Dew Can.

Notice that the depth image of the object doesn't rotate at all: the object rotates at the environment, but the viewpoint of the Kinect remains still.

The dataset is not split into training and testing instances, thus the classification step used cross-validation (10-fold).

$1 \quad$ At https://rgbd-dataset.cs.washington.edu/ - Accessed May 29, 2017 

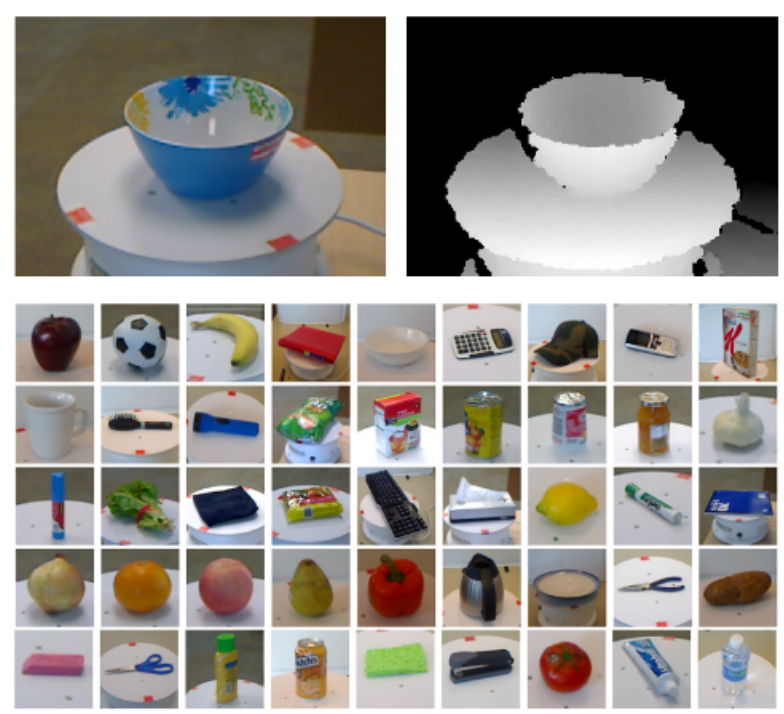

Figure 31 - University of Washington UW RGB-D Object Dataset. On the top: object captured from UW dataset. On the bottom: subset of objects from UW dataset. Source: Lai et al. (2011). Copyright (C) 2011, IEEE.

\subsection{Princeton's ModelNet Dataset}

The ModelNet Dataset (WU et al., 2015), from Princeton, contains meshes from several datasets and benchmarks. The number of instances is not the same for each category: for instance, aircraft objects have much more instances than the X-Box category.

Figure 32 contains examples from eight classes of the MN-DS.

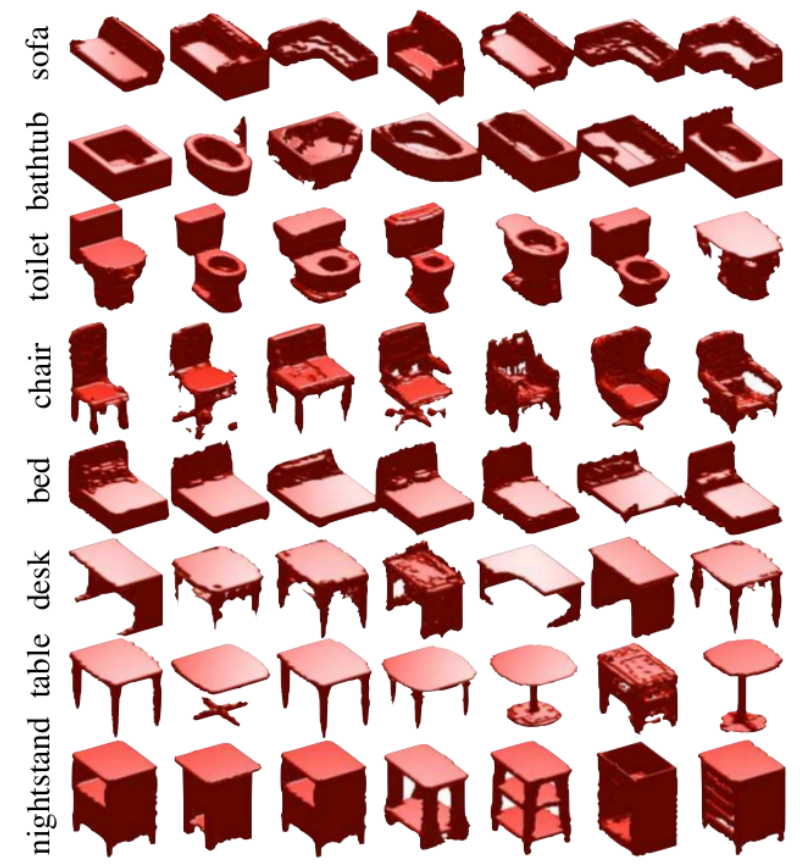

Figure 32 - Samples from the MN-DS. Source: Wu et al. (2015). Copyright (c) 2015, IEEE. 
another with all 40 object classes (MN-40). The proposed method was tested on the MN-10 subset.

\subsection{DNet}

The 3DNet, presented by Wohlkinger et al. (2012), is a hierarchical models dataset, in which the training data is a set of mesh models, while the test data is a set of RGB-D scenes that contain only the object. The dataset contains several subsets with increasing difficulty. They are:

- Cat10: contains 10 classes of object shapes. The objects have a little similarity on their shapes, but they are still distinct.

- Cat50: contains, in addiction to the Cat10 classes, more 50 classes of object shapes. However, the objects classes are also divided into sub-categories (chair, arm-chair or office-chair, for instance).

- Cat 100: contains 100 classes of objects shapes with color information. It contains shapes so similar that the instances can only be uniquely distinguished when considering their color.

- Cat200: contains 200 classes of objects shapes with color information. However, to classify the shapes in this subset it is important to consider the objects' size.

For this work's purpose, the Cat10 subset was employed to test the efficiency of the method to classify classes of objects that have substantially different shapes. However, as the test set contains RGB-D scenes in which the objects must be detected for pose estimation (without segmentation), the dataset was trained and tested using only the training instances of the classes.

\subsection{Dataset Comparison}

Occlusion. The first aspect to be noticed between the datasets is that the UW dataset provides depth information of a scene, which means that the object was observed from the sensor from a determined viewpoint, thus everything behind the object in respect to the viewpoint is not present on the 3D Point Cloud, while the objects from the MN-DS and from the 3DNet training set have no occluded parts. The Figure 33 illustrates these differences.

Class Variation. The UW-DS instances from each class are similar in shape. For instance: all the instances of the class "Apple" are similar in shape. The same applies to the 3DNet. On the other hand, the shapes from each instance of the MN-DS classes vary inside the class. An example is the class of bathtubs, represented on the Figure 34. The shapes of the bathtubs change drastically. 

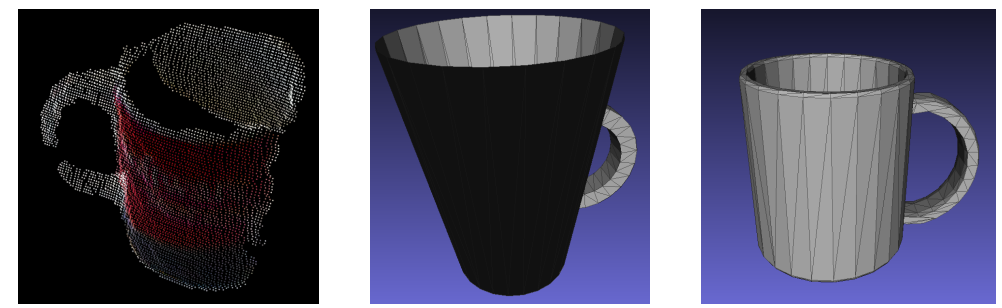

Figure 33 - Mugs represented on different data representation. From the left to the right: an UW segmented RGB-D data; 3D mesh from the MN model; and 3D mesh from the 3DNet train set.
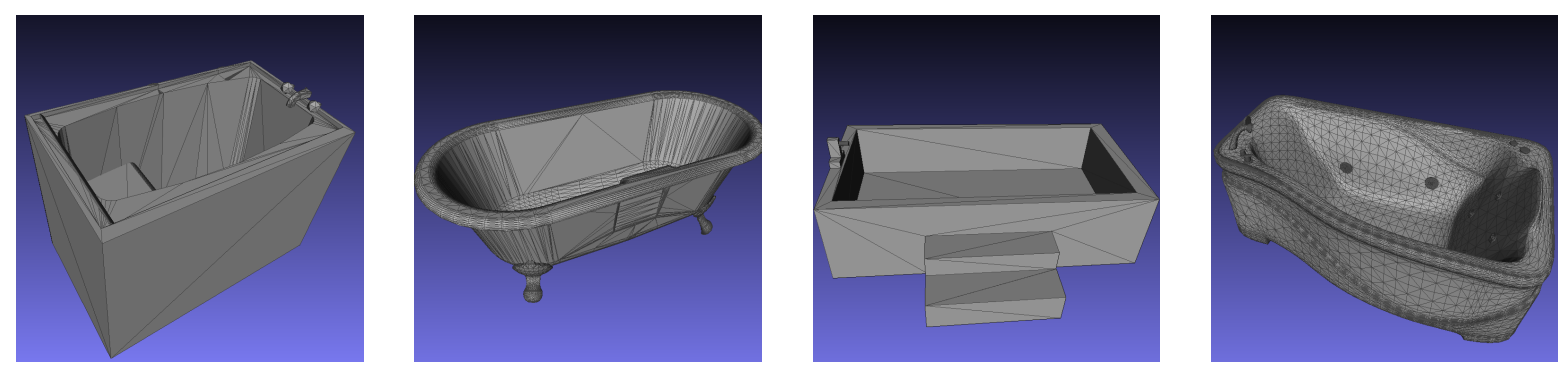

Figure 34 - Differences between instances inside the class bathtub.

Data Structures. The data structure employed to represent the objects is different on the datasets: the UW dataset represents its objects on point clouds; the MN dataset and the 3DNet train set are represented by meshes. Since meshes need to be rendered to find all the points for each face, this could lead to data/shape loss. 
CHAPTER

8

VALIDATION STRATEGY

The proposed methods have theoretical foundation that support them. However, how do they behave when tested?

This chapter presents how the tests were prepared for each method. In the following sections details of the datasets preparation, the parameters that were used and how data were classified are presented. The classifier used in all tests was the k-Nearest Neighbors (kNN) algorithm.

\subsection{COMSD-3D}

\subsubsection{Dataset Setup}

In 3D computer vision context, COMSD is a method that extracts features from cooccurrence matrices computed from voxel grids. Since the dataset used to perform the tests represent their objects in an RGB-D point cloud format, a conversion is needed from point cloud to a binary voxel grid. Since it is a binary voxel grid, given the integer indexes $i, j$ and $k$ (row, column and depth, respectively), the voxel $V$ is filled $(V[i, j, k]=1)$ if any point from the point cloud belongs on that position. Otherwise, $V[i, j, k]=0$. Furthermore, all color information is removed on the conversion.

Given an empty voxel grid $V$ (empty in this case means that all of its voxels are filled with 0 ) and a $3 \mathrm{D}$ point cloud $C$, the conversion of each point $P$ on the coordinates $(x, y, z)$ is 


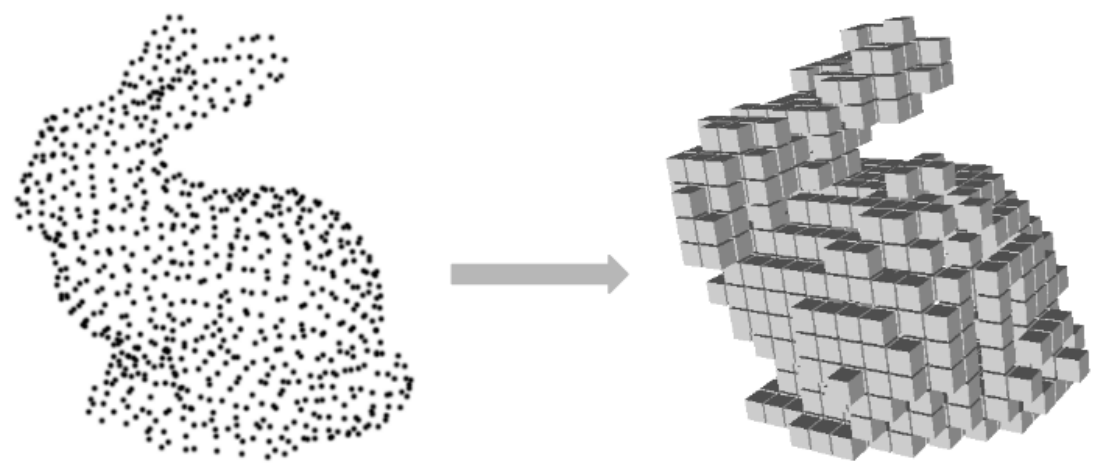

Figure 35 - The conversion from the 3D point cloud representation of the Stanford bunny (left) to a voxel grid (right).

performed as follows:

$$
\begin{aligned}
i & =\left\lfloor\frac{y-y_{\min }}{y_{\max }-y_{\min }} \cdot(H-1)\right\rfloor \\
j & =\left\lfloor\frac{x-x_{\min }}{x_{\max }-x_{\min }} \cdot(W-1)\right\rfloor \\
k & =\left\lfloor\frac{z-z_{\min }}{z_{\max }-z_{\min }} \cdot(D-1)\right\rfloor
\end{aligned}
$$

and

$$
V[i, j, k]=1
$$

where $x_{\min }, y_{\min }, z_{\min }, x_{\max }, y_{\max }, z_{\max }$ are extrema point coordinates from $C ; H, W$ and $D$ are the voxel grid dimensions height, width and depth, respectively. Figure 35 represents a conversion from a $3 \mathrm{D}$ point cloud to voxel grids. This conversion ensures the scale invariance of the method.

\subsubsection{Defined Parameters}

To evaluate the proposed method, the features were computed for each object instance on the dataset and labeled with the class of the object. The only parameter changed for each test was $N$, the dimensions of each voxel grid. After the extraction of the features, the classification step was done with the $\mathrm{kNN}(k=1,3,5)$ machine learning algorithm with cross validation (10-fold) using the Weka Tool ${ }^{1}$ - Waikato Environment for Knowledge Analysis. Weka is a well known Open Source Data Mining Software available in Java.

\subsection{CNSD}

To evaluate the CNSD method, the features were computed from each object instance of the UW dataset and were labeled with the class of the object. 
Since an adjacency matrix stores $N^{2}$ values, where $N$ is the size of the point cloud, each object point cloud of the dataset had to be downsampled. This was a necessary step because either there was no memory available to process the whole adjacency matrix from an point cloud or it took too long to extract features a single point cloud.

Two different techniques for point cloud downsampling were applied:

- A Box Grid Filtering (BGF), which basically consists in converting the point cloud to a voxel grid and then converting it back to point clouds. This reduction technique was used only on clouds larger than 8000 points to a size of 7000 to 8000 ;

- And Random Points Removal (RPF), which removes randomly $60 \%$ of each cloud. This technique was used to test how robust to erosion the method is.

After the feature extraction process, classification was performed using the k-NN algorithm available on the Weka Tool.

\subsection{RPHSD}

\subsubsection{Dataset Setup}

To execute the method, some adaptations needed to be made at the input data for the three datasets.

UW Dataset. The input point clouds were loaded discarding RGB information.

MN Dataset. The dataset was converted from mesh to point cloud data structure. However, the dataset was at the Object File Format (OFF), which has no known converters for the authors. Then, a converter named off $2 p c d$ was developed and uploaded to the project's Github repository with a script that automates the whole MN-DS conversion. The converter loads to a point cloud all vertices and samples the edge and surface triangles points from the mesh. The sampling of the edges' points consists in plotting the points between the line that connects the two vertices from the edge. To sample the surface triangles, other lines were sampled between each point of two different edges of the polygon. After sampling the mesh to a point cloud, this is downsampled using a voxel grid filter.

3DNet. It was necessary to convert the mesh instances to point cloud. Therefore, the software mesh2pcd, available on the Point Cloud Library tools, was used to convert the instances using its default parameters (level $=2$, resolution $=100^{\circ}$ and leaf_size $=0.01$ ).

All. The datasets (converted to point cloud format) had their points coordinates translated to the cloud mass center. After, the clouds were normalized to range between -1 and 1 (using 

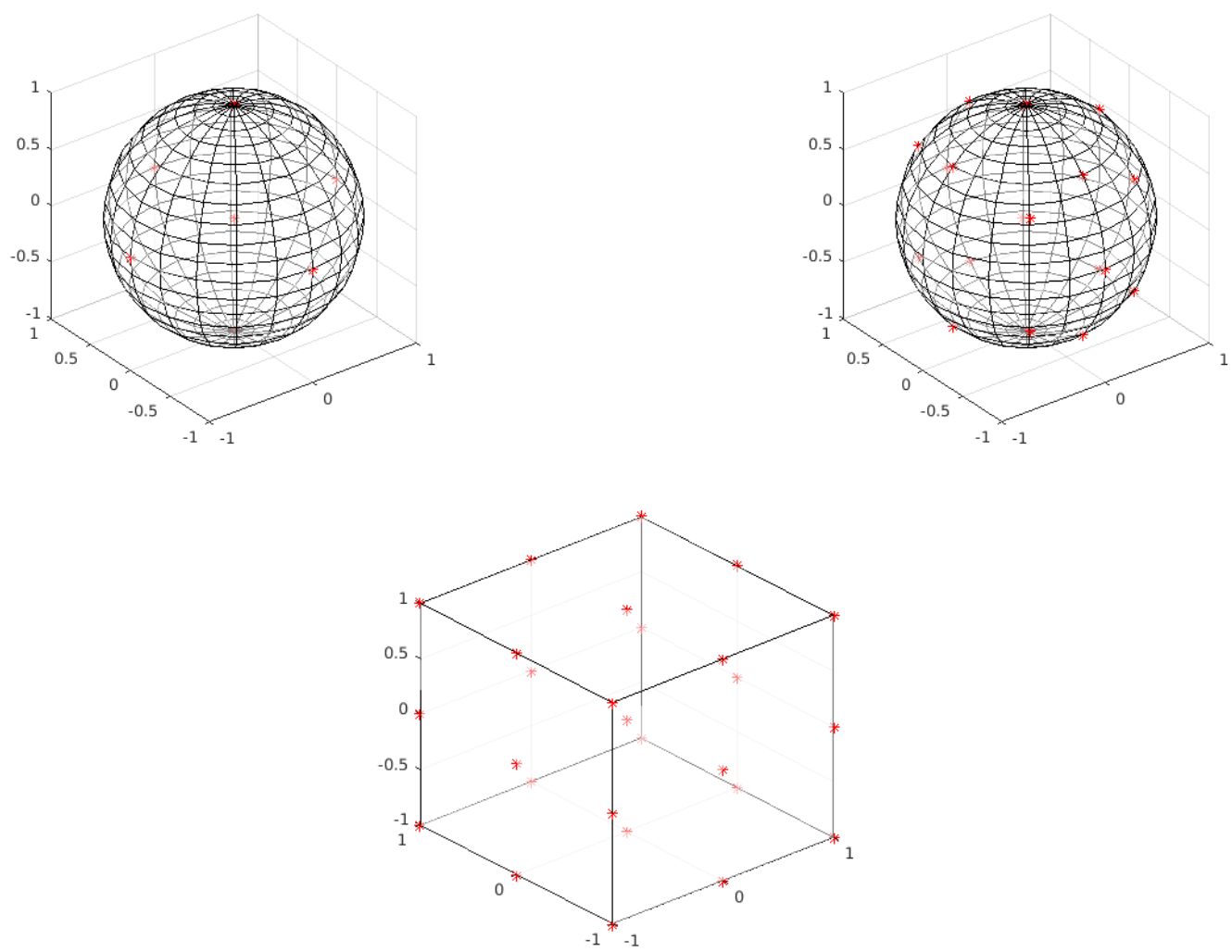

Figure 36 - The reference points arrangements. From the left to the right: $A_{1}$ cross; $A_{2}$ spherical; and $A_{3}$ cubic .

the Equation 8.3).

$$
p_{i}^{\prime}=\frac{p_{i}}{\max _{\left(\left|\max _{p}\right|,\left|\min _{p}\right|\right)}}
$$

where $p$ is the point before the normalization and $p^{\prime}$ is the point after the normalization; $i$ is the coordinate $x, y$ or $z$ of the point; $\max \left(\left|\max _{p}\right|,\left|\min _{p}\right|\right)$ selects the maximum between the maximum $\max _{p}$ and the minimum $\min _{p}$ coordinates values of the cloud.

\subsubsection{Defined Parameters}

The $\mathrm{kNN}$ was applied to classify the extracted features with $k=1,5,9$. The variable parameters for both experiments were: the size of the histograms $S_{1}=10, S_{2}=30, S_{3}=50$, $S_{4}=70$ and $S_{5}=100$; different arrangements and number of reference points $A_{1}=7$ points (cross arrangement), $A_{2}=27$ points (spherical arrangement) and $A_{3}=27$ points (cubic arrangement), arranged as in Figure 36; and tests with the reduced and the complete descriptors set were made. Furthermore, cross-validation was used on the classification of the UW-DS (10-fold) and the 3DNet (5-fold) instances. 
CHAPTER

\subsection{Accuracy}

The accuracy of the COMSD-3D method for the UW-DS is shown in Table 3, of the CNSD-3D are in Table 4. The best results yielded by the experiments of the RPHSD are displayed in Table 5.

\begin{tabular}{c|c|c|c|c}
$k$ & $N=30$ & $N=50$ & $N=75$ & $N=100$ \\
\hline 1 & $\mathbf{8 2 . 1} \pm \mathbf{0 . 3}$ & $\mathbf{8 2 . 3} \pm \mathbf{0 . 2}$ & $\mathbf{8 1 . 3} \pm \mathbf{0 . 2}$ & $73.9 \pm 0.3$ \\
3 & $80.8 \pm 0.3$ & $81.1 \pm 0.2$ & $80.6 \pm 0.2$ & $73.9 \pm 0.3$ \\
5 & $80.2 \pm 0.3$ & $80.5 \pm 0.2$ & $80.1 \pm 0.2$ & $\mathbf{7 4 . 1} \pm \mathbf{0 . 3}$
\end{tabular}

Table 3 - COMSD-3D accuracy(\%) classified with kNN on the UW-DS.

\begin{tabular}{c|c|c|c} 
Downsample Method & $1-\mathrm{NN}$ & $5-\mathrm{NN}$ & $15-\mathrm{NN}$ \\
\hline BGF & $\mathbf{7 9 . 6 8} \pm$ n.d. & $77.95 \pm$ n.d. & $74.63 \pm$ n.d. \\
RPR & $72.01 \pm$ n.d. & $\mathbf{7 2 . 4 0} \pm$ n.d. & $70.67 \pm$ n.d.
\end{tabular}

Table 4 - CNSD-3D accuracy(\%) classified with k-NN, with K equals to 1, 5 and 15 respectively on the UW-DS. Standard deviation values missing (n.d. - not defined) due to the default Weka Interface results.

\subsection{Comparison}

This subsection presents a comparison of the obtained scores with previous works obtained on the UW and the MN datasets. Since the validation method of the 3DNet is different from the method used in previous works, the methods were not comparable and then no comparison was made using this dataset.

UW-DS. The work from Cheng Xin Zhao (2016) provides a comparison between their method accuracy and the most relevant methods accuracy applied to the UW-DS for object 


\begin{tabular}{c|c|c|c|c|c} 
Dataset & Descriptor Type & Histogram Size & Arrange & $\mathrm{K}$ & Accuracy \\
\hline UW-DS & reduced & 10 & $A_{2}$ & 9 & $39 \pm 2$ \\
UW-DS & complete & 100 & $A_{3}$ & 1 & $\mathbf{8 5} \pm \mathbf{3}$ \\
MN-DS & reduced & 10 & $A_{1}$ & 1 & 53 \\
MN-DS & complete & 100 & $A_{3}$ & 1 & $\mathbf{8 1}$ \\
3DNet & reduced & 10 & $A_{2}$ & 1 & $77 \pm 3$ \\
3DNet & complete & 70 & $A_{3}$ & 1 & $\mathbf{9 7} \pm \mathbf{3}$
\end{tabular}

Table 5 - RPHSD best accuracy (\%) results for each dataset. The Descriptor Type to Arrange columns are the parameters used on the method. The $K$ column represents the $k$ parameter of the kNN algorithm.

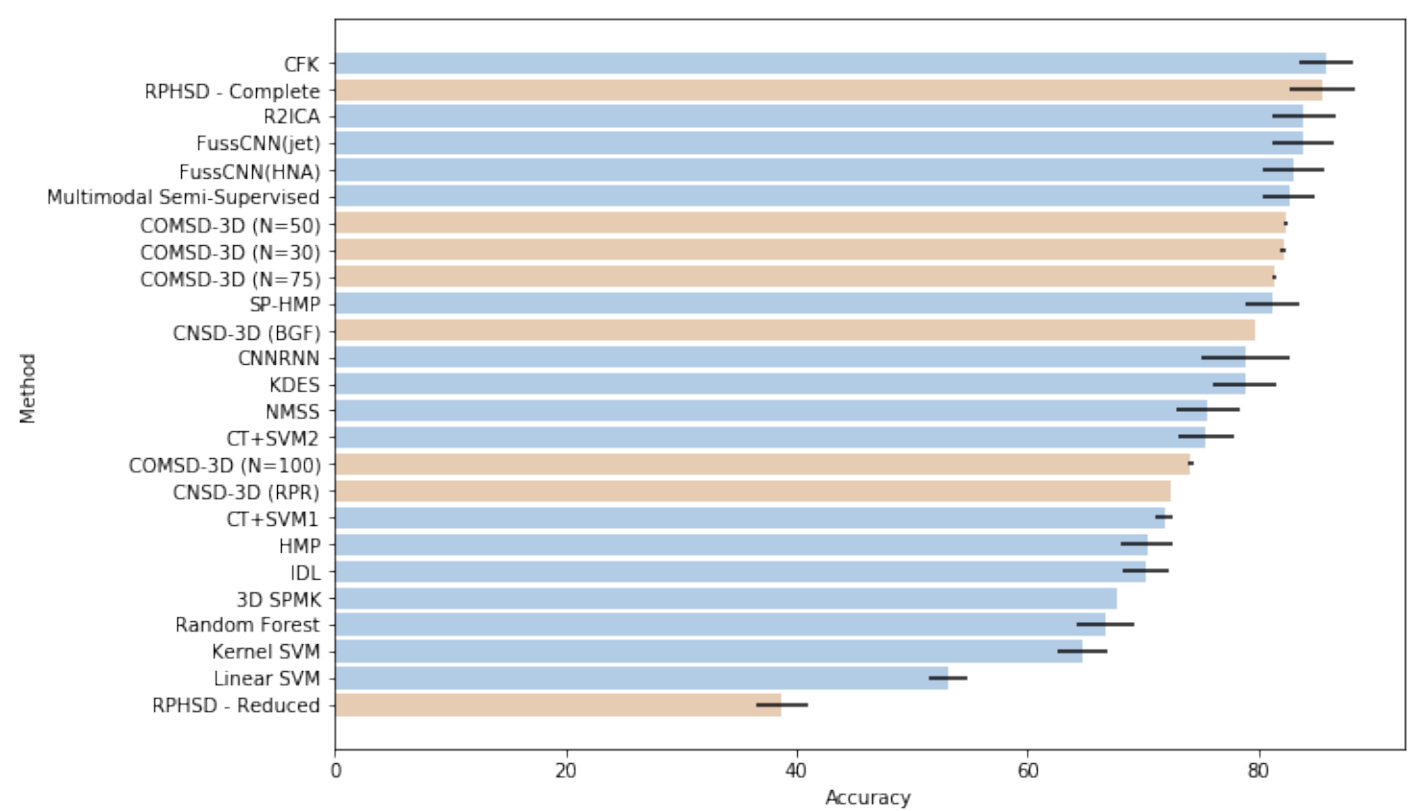

Figure 37 - Bar chart of recent works performance for object classification on UW objects dataset. The brown bars represent the results for the proposed methods. The blue bars are the other methods results and the black lines are the deviance of each method accuracy.

classification. Their comparison considers the results using either depth information (3D), color information (RGB) or using both. In this work, the comparison was made only with the results using depth information. In the Table 6 the best results obtained from the proposed methods (bold font) were compared with the others presented on the comparison table obtained from Cheng Xin Zhao (2016). The Figure 37 illustrates the performance of our proposed method, among the other methods performance. More details of the other methods considered in the Figure 37 can be found on Cheng Xin Zhao (2016).

MN-DS. On the web page of the MN-DS ${ }^{1}$, a table with the scores presented on works which classified the objects from the dataset is shown. The Table 7 presents the previous scores from MN 10 with the results from this method. Also, Figure 38 provide a visualization of this method results position among the results from Table 7 .

$1 \quad$ At: http://modelnet.cs.princeton.edu/ 


\begin{tabular}{|c|c|c|}
\hline Method & Mean Accuracy & Standard Deviance \\
\hline Linear SVM & 53.1 & 1.7 \\
\hline Kernel SVM & 64.7 & 2.2 \\
\hline Random Forest & 66.8 & 2.5 \\
\hline IDL & 70.2 & 2.0 \\
\hline 3D SPMK & 67.8 & - \\
\hline KDES & 78.8 & 2.7 \\
\hline HMP & 70.3 & 2.2 \\
\hline SP-HMP & 81.2 & 2.3 \\
\hline CNNRNN & 78.9 & 3.8 \\
\hline R2ICA & 83.9 & 2.8 \\
\hline FussCNN(HNA) & 83.0 & 2.7 \\
\hline FussCNN(jet) & 83.8 & 2.7 \\
\hline NMSS & 75.6 & 2.7 \\
\hline CFK & 85.8 & 2.3 \\
\hline $\mathrm{CT}+\mathrm{SVM} 1$ & 71.8 & 0.8 \\
\hline $\mathrm{CT}+\mathrm{SVM} 2$ & 75.4 & 2.4 \\
\hline Multimodal Semi-Supervised & 82.6 & 2.3 \\
\hline COMSD-3D $(\mathrm{N}=30)$ & 82.1 & 0.3 \\
\hline COMSD-3D $(\mathrm{N}=50)$ & 82.3 & 0.2 \\
\hline COMSD-3D $(\mathrm{N}=75)$ & 81.3 & 0.2 \\
\hline COMSD-3D $(\mathrm{N}=100)$ & 74.1 & 0.3 \\
\hline CNSD-3D (BGF) & 79.7 & n.d. \\
\hline CNSD-3D (RPR) & 72.4 & n.d. \\
\hline RPHSD - Reduced & 38.7 & 2.2 \\
\hline RPHSD - Complete & 85.4 & 2.8 \\
\hline
\end{tabular}

Table 6 - Comparison between the presented methods and recent results on UW dataset. The result in blue is the best comparable result from the previous works. The results obtained in this work are in bold font.

\subsection{Results Analysis}

\subsubsection{COM3D-SD}

From the experimental results presented in Table 6 and Figure 37, it is possible to conclude that the COMSD-3D yields an accuracy of $82.3 \pm 0.2 \%$, being among the best results obtained on the UW-DS. Other methods achieved higher accuracies ranging between 82.6 and $85.8(82.6,83.0,83.8,83.9$ and 85.8 , respectively). It is important to consider that these methods have a higher standard deviation over their mean accuracy results, which means that we can consider our method as good as these others. Only the CFK method showed a relative more significant performance over these top performance algorithms. 


\begin{tabular}{c|c} 
Method & MN 10 Accuracy $(\%)$ \\
\hline ECC & 90.0 \\
PANORAMA-NN & 91.1 \\
Klokov and Lempitsky & 94.0 \\
LightNet & 93.39 \\
Xu and Todorovic & 88.00 \\
Geometry Image & 88.4 \\
PointNet & 77.6 \\
3D-GAN & 91.0 \\
VRN Ensemble & 97.14 \\
ORION & 93.8 \\
FusionNet & 93.11 \\
Pairwise & 92.8 \\
GIFT & 92.35 \\
VoxNet & 92 \\
DeepPano & 85.45 \\
3DShapeNets & 83.5 \\
RPHSD - Reduced & $\mathbf{5 2 . 9 2}$ \\
RPHSD - Complete & $\mathbf{8 1 . 2 6}$
\end{tabular}

Table 7 - Comparison between this work and recent results on MN dataset. The result in blue is the best comparable result from the previous works. The results obtained in this work are in bold font.

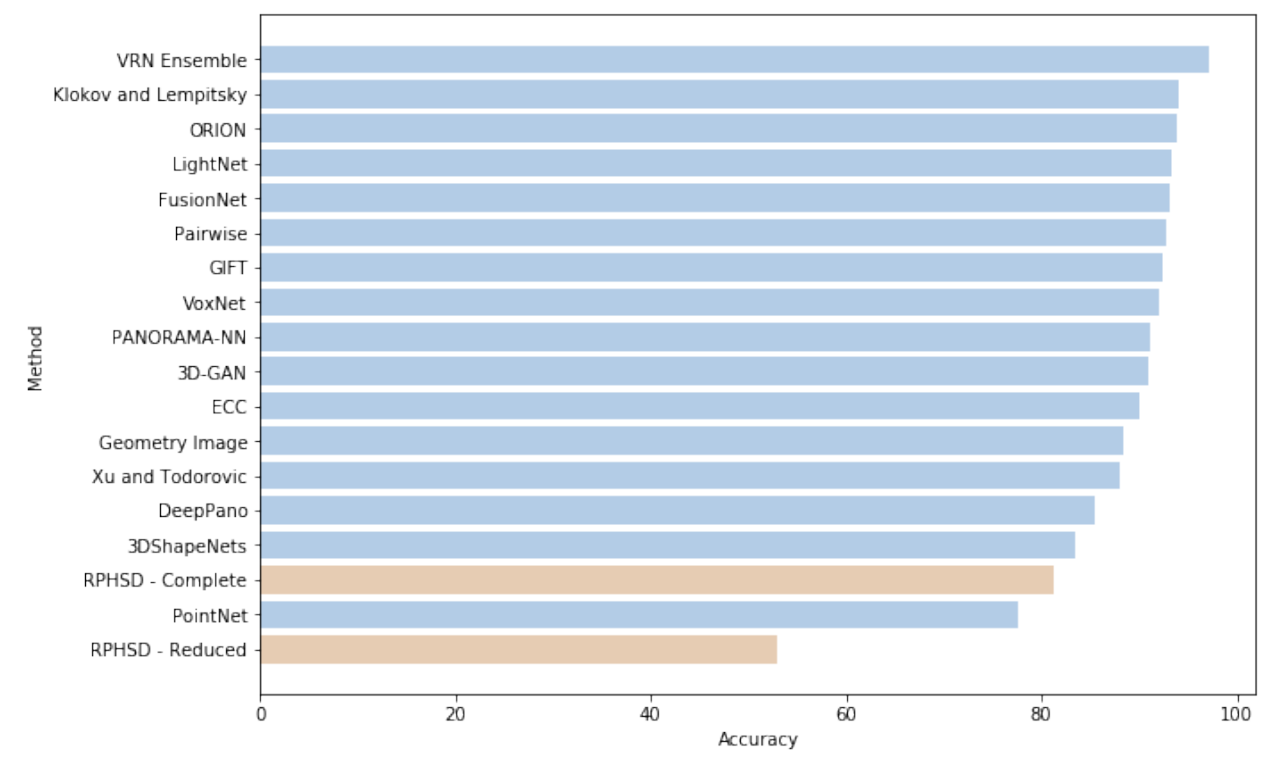

Figure 38 - Bar chart of recent works performance for object classification on the MN dataset. The brown bars represent this work results, the blue bars are the other methods results. 


\subsubsection{CNSD-3D}

As observed in Table 6 and Figure 37, the CNSD-3D can reach an accuracy of 79.7\%, being in the seventh place, among the best results based on the use of this dataset. The other methods that are better than the proposed method have an accuracy that ranges from $81.2 \%$ to $85.8 \%$. It is important to notice that the proposed method was presented in its first proposed version, adopting this new Complex Networks based algorithm as the feature extractor used to recognize 3D shapes on the UW RGB-D Object Dataset. However, since the aim of the tests with that technique was to prove that the complex networks could be used to extract competitive $3 \mathrm{D}$ features, there was no investment on sophisticated machine learning techniques. On the other hand, an increase in the classification accuracy could be achieved using more elaborated techniques as neural networks and random forests.

\subsubsection{RPHSD}

The results displayed in this section show a huge difference in accuracy between the reduced descriptor and the complete descriptor: the reduced descriptor, despite being - theoretically - rotation invariant, has a lower accuracy than the complete descriptor. Either: (i) the number of reference points is relatively small, considering that the angle between the points are high, (ii) the reference points arrangements have non-uniform spaced points, which is not in respect to the conditions from the Section 6.2, or (iii) the descriptor may be too small to describe properly the input shapes.

Also, the difference of the results on the datasets may lead us to the major conclusion about the descriptor: it is sensitive to shape variation at the same class of objects.

Despite the presented observations, the method is still competitive: it matches the performance of the state-of-the-art methods.

\subsection{Overview}

COMSD-3D. The COMSD-3D method, which consists in the conversion and adjustment of the provided data into the parameterized voxel grid, generation of the SDM, selection of a set of statistical features, and application of the $\mathrm{kNN}$ method for classification, demonstrated that is a solution that can be competitive in terms of accuracy compared to other methods in a $O(N+k \cdot l \cdot m)$ complexity $^{2}$.

CNSD-3D. The CNSD-3D approach, with complexity ${ }^{3}$ of $O\left(N^{2}\right)$, based on complex networks data analysis and feature extraction, together with a machine learning algorithm (k-NN),

$2 N$ is the size of the point cloud; $k, l$ and $m$ are the number of voxel grid rows, columns and depth size.

$3 \quad N$ is the size of the point cloud. 
also demonstrated that, even applied on such degraded and downsampled input, is a solution that can be competitive in terms of accuracy compared to other methods found in the literature.

RPHSD. The RPHSD, which computes histograms of distances from reference points to the point cloud, showed a considerable accuracy: it separated well the classes from the 3DNet (97\% of accuracy) and stood among the best methods (in terms of accuracy) of the UW-DS rank in a complexity ${ }^{4}$ of $O(k \cdot N)$.

$\overline{4} k$ is the number of reference points and $N$ is the size of the point cloud. 
CHAPTER

10

CONCLUSION

In this project, novel methods (COMSD-3D, CNSD-3D and RPHSD) were introduced and tools were developed. With two of these methods, two papers were submitted and accepted.

The COMSD-3D and the CNSD are adaptations of the traditional techniques: the cooccurrence matrices in computer vision, originally, were used only applied to compute textures. In our paper (PRZEWODOWSKI FILHO; OSÓRIO, 2017a) we demonstrated that it is possible - and effective - to use their features to describe 3D shapes. Also, the Complex Networks applications were restricted only to 2D images until we presented their application on 3D point clouds with promising expansion and improvement (PRZEWODOWSKI FILHO; OSÓRIO, 2017b).

The RPHSD method is a novel efficient method to describe shapes. Experimental results have shown this method matches the performance of state-of-art methods tested at the same benchmark datasets. However, it showed better performance on datasets with small intra-class variation in shape. Another remarkable feature of this method is the computational speed: its complexity is relatively low and can be employed on real-time applications, mainly in robotics, such as autonomous vehicles, landmarks for mobile robots and industry robots. Also, it has few and easy-to-understand parameters: the set of reference points positions, a point cloud and the size of each histogram (or the inverse of the step size).

Finally, further research could lead to:

- COMSD-3D. Parameters optimization and performance improvement using other voxel data structures than the voxel grids;

- CNSD-3D. New features for the CNSD-3D that could lead to higher classification accuracy;

- RPHSD. 
- Proposal of new features to be computed from the beyond the entropy, variance and inverse difference moment and other ways of selecting reference points;

- Feature extraction in other dimensional signals.

- Classifiers. A detailed study on other machine learning techniques could define a proper classifier for each descriptor of the proposed methods, which could improve the accuracy of the classification step. 


\section{BIBLIOGRAPHY}

BACKES, A. R.; CASANOVA, D.; BRUNO, O. M. A complex network-based approach for boundary shape analysis. Pattern Recognition, p. 54-67, 2009. Citations on pages 47, 48, and 49.

Texture analysis and classification: A complex network-based approach. Information Sciences, p. 168-180, 2013. Citation on page 47.

BACKES, A. R.; MARTINEZ, A. S.; BRUNO, O. M. Texture analysis using graphs generated by deterministic partially self-avoiding walks. Pattern Recognition, p. 1684-1689, 2011. Citation on page 47 .

CHENG XIN ZHAO, R. C. Z. L. K. H. Y. R. Y. Semi-Supervised Multimodal Deep Learning for RGB-D Object Recognition. In: Proceedings of the Twenty-Fifth International Joint Conference on Artificial Intelligence (IJCAI-16) Semi-Supervised. [S.1.: s.n.], 2016. p. 33453351. Citations on pages 71 and 72.

CHENG, Y.; CAI, R.; ZHAO, X.; HUANG, K. Convolutional Fisher Kernels for RGB-D Object Recognition. In: Proceedings - 2015 International Conference on 3D Vision, 3DV 2015. [S.1.: s.n.], 2015. p. 135-143. ISBN 9781467383325. Citations on pages 12 and 39.

DARAS, P.; ZARPALAS, D.; TZOVARAS, D.; STRINTZIS, M. G. 3D Model search and retrieval based on the spherical trace transform. 2004. 335-338 p. Citations on pages 11, 32 , and 33.

Efficient 3-D model search and retrieval using generalized 3-D radon transforms. IEEE Transactions on Multimedia, p. 101-114, 2006. Citations on pages 11, 33, and 34.

DROST, B.; ILIC, S. 3D Object Detection and Localization Using Multimodal Point Pair Features. In: 2012 Second International Conference on 3D Imaging, Modeling, Processing, Visualization \& Transmission. [S.1.: s.n.], 2012. p. 9-16. ISBN 978-0-7695-4873-9. Citations on pages 11 and 36.

EITEL, A.; SPRINGENBERG, J. T.; SPINELLO, L.; RIEDMILLER, M.; BURGARD, W. Multimodal deep learning for robust RGB-D object recognition. In: IEEE International Conference on Intelligent Robots and Systems. [S.1.: s.n.], 2015. p. 681-687. ISBN 9781479999941. Citation on page 40 .

FLORINDO, J. B.; LANDINI, G.; BRUNO, O. M. Three-dimensional connectivity index for texture recognition. Pattern Recognition Letters, p. 239-244, 2016. Citation on page 48.

GEBHARDT, S.; PAYZER, E.; SALEMANN, L.; FETTINGER, A.; ROTENBERG, E.; SEHER, C. Polygons, Point-Clouds , and Voxels , a Comparison of High-Fidelity Terrain Representations. Fall Simulation Interoperability Workshop, p. 1-9, 2009. Citations on pages 11, 26, 27, and 28 . 
GONÇALVES, W. N.; BRUNO, O. M. Dynamic texture analysis and segmentation using deterministic partially self-Avoiding walks. Expert Systems with Applications, p. 4283-4300, 2013. Citation on page 48.

HARALICK, R. M.; SHANMUGAM, K. Textural Features for Image Classification. IEEE Transactions on Systems, Man, and Cybernetics, p. 610-621, 1973. Citations on pages 41 and 44.

JHUO, I.-H.; GAO, S.; ZHUANG, L.; LEE, D.; MA, Y. Unsupervised feature learning for RGB-D image classification. [S.1.: s.n.], 2015. 276-289 p. ISBN 9783319168647. Citation on page 39 .

KADYROV, A.; PETROU, M. The trace transform and its applications. IEEE Transactions on Pattern Analysis and Machine Intelligence, p. 811-828, 2001. Citations on pages 11, 31, and 32 .

KURANI, A.; XU, D.; FURST, J.; RAICU, D. Co-occurrence matrices for volumetric data. 7th IASTED International Conference on Computer Graphics and Imaging, p. 447-452, 2004. Citations on pages 43 and 44 .

LAI, K.; BO, L.; REN, X.; FOX, D. A large-scale hierarchical multi-view RGB-D object dataset. In: Proceedings - IEEE International Conference on Robotics and Automation. [S.1.: s.n.], 2011. p. 1817-1824. ISBN 9781612843865. Citations on pages 12, 63, and 64.

LLOYD, K.; ROSIN, P. L.; MARSHALL, D.; MOORE, S. C. Detecting violent and abnormal crowd activity using temporal analysis of grey level co-occurrence matrix (GLCM)-based texture measures. Machine Vision and Applications, p. 361-371, 2017. Citation on page 41.

MAHMOUD-GHONEIM, D.; TOUSSAINT, G.; CONSTANS, J. M.; CERTAINES, J. D. D. Three dimensional texture analysis in MRI: A preliminary evaluation in gliomas. Magnetic Resonance Imaging, p. 983-987, 2003. Citation on page 43.

MATURANA, D.; SCHERER, S. VoxNet: A 3D Convolutional Neural Network for real-time object recognition. In: 2015 IEEE/RSJ International Conference on Intelligent Robots and Systems (IROS). [S.1.: s.n.], 2015. p. 922-928. ISBN 978-1-4799-9994-1. Citations on pages 12 and 38.

OTHMEN, E. B.; SAYADI, M.; FNIAECH, F. 3D gray level co-occurrence matrices for volumetric texture classification. In: 2013 3rd International Conference on Systems and Control, ICSC 2013. [S.1.: s.n.], 2013. p. 833-837. ISBN 978-1-4799-0275-0. Citations on pages 15, 41, and 43.

PRZEWODOWSKI FILHO, C. A. B.; OSÓRIO, F. S. Co-occurrence matrices for 3d shape classification. In: 2017 Latin American Robotics Symposium (LARS) and 2017 Brazilian Symposium on Robotics (SBR). [S.1.: s.n.], 2017. p. 1-5. Citations on pages 42 and 77.

Complex network shape descriptor for 3d objects classification. In: 2017 Latin American Robotics Symposium (LARS) and 2017 Brazilian Symposium on Robotics (SBR). [S.1.: s.n.], 2017. p. 1-5. Citations on pages 48 and 77.

RUSU, R. B.; BLODOW, N.; BEETZ, M. Fast Point Feature Histograms (FPFH) for 3D registration. In: IEEE International Conference on Robotics and Automation. [S.1.: s.n.], 2009. p. 3212-3217. ISBN 978-1-4244-2788-8. Citations on pages 11, 34, and 35. 
RUSU, R. B.; MARTON, Z. C.; BLODOW, N.; BEETZ, M. Learning informative point classes for the acquisition of object model maps. In: 2008 10th International Conference on Control, Automation, Robotics and Vision, ICARCV 2008. [S.1.: s.n.], 2008. p. 643-650. ISBN 9781424422876. Citation on page 34 .

SAMPSON, D. J.; CHANG, Y. K.; RUPASINGHE, H. P. V.; ZAMAN, Q. U. A dual-view computer-vision system for volume and image texture analysis in multiple apple slices drying. Journal of Food Engineering, p. 49-57, 2014. Citation on page 41.

STEDER, B.; RUSU, R. B.; KONOLIGE, K.; BURGARD, W. NARF: 3D Range Image Features for Object Recognition. October, 2010. Citation on page 37.

Point feature extraction on 3D range scans taking into account object boundaries. In: Proceedings - IEEE International Conference on Robotics and Automation. [S.l.: s.n.], 2011. p. 2601-2608. ISBN 9781612843865. Citation on page 37.

TANG, J.; JIANG, B.; CHANG, C.-C.; LUO, B. Graph structure analysis based on complex network. Digital Signal Processing, p. 713-725, 2012. Citation on page 47.

WOHLKINGER, W.; ALDOMA, A.; RUSU, R. B.; VINCZE, M. 3DNet: Large-scale object class recognition from CAD models. In: Proceedings - IEEE International Conference on Robotics and Automation. [S.1.: s.n.], 2012. p. 5384-5391. ISBN 9781467314039. Citation on page 65.

WU, Z.; SONG, S.; KHOSLA, A.; YU, F.; ZHANG, L.; TANG, X.; XIAO, J. 3D ShapeNets: A deep representation for volumetric shapes. In: Proceedings of the IEEE Computer Society Conference on Computer Vision and Pattern Recognition. [S.1.: s.n.], 2015. p. 1912-1920. ISBN 9781467369640. Citations on pages 12 and 64.

YAHIA, S.; SALEM, Y. B.; ABDELKRIM, M. N. 3D face recognition using local binary pattern and grey level co-occurrence matrix. In: 17th International Conference on Sciences and Techniques of Automatic Control and Computer Engineering (STA). Sousse, Tunisia: IEEE, 2016. p. 328-338. Citation on page 41. 


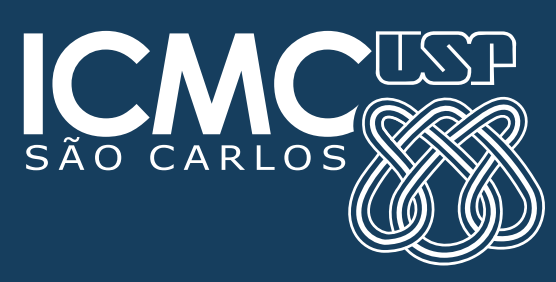

